\title{
Djankuat glacier station in the North Caucasus, Russia: a database of glaciological, hydrological, and meteorological observations and stable isotope sampling results during 2007-2017
}

\author{
Ekaterina P. Rets ${ }^{1}$, Viktor V. Popovnin ${ }^{2}$, Pavel A. Toropov ${ }^{2,9}$, Andrew M. Smirnov ${ }^{3}$, Igor V. Tokarev ${ }^{4}$, \\ Julia N. Chizhova ${ }^{5}$, Nadine A. Budantseva ${ }^{2}$, Yurij K. Vasil'chuk ${ }^{2}$, Maria B. Kireeva ${ }^{2}$, \\ Alexey A. Ekaykin ${ }^{6,7}$, Arina N. Veres ${ }^{6}$, Alexander A. Aleynikov ${ }^{2}$, Natalia L. Frolova ${ }^{2}$, \\ Anatoly S. Tsyplenkov ${ }^{2}$, Aleksei A. Poliukhov ${ }^{2,8}$, Sergey R. Chalov ${ }^{2}$, Maria A. Aleshina ${ }^{9}$, and \\ Ekaterina D. Kornilova ${ }^{2}$ \\ ${ }^{1}$ Water Problems Institute, Russian Academy of Sciences, Moscow, 119333, Russia \\ ${ }^{2}$ Faculty of Geography, Lomonosov Moscow State University, Moscow, 119991, Russia \\ ${ }^{3}$ Laboratory of Glaciology, Institute of Geography, Russian Academy of Sciences, Moscow, 119017, Russia \\ ${ }^{4}$ St. Petersburg State University, Geomodel Resource Center, St. Petersburg, 199034, Russia \\ ${ }^{5}$ Institute of Geology of Ore Deposits, Petrography, Mineralogy and Geochemistry, \\ Russian Academy of Science, Moscow, 109017, Russia \\ ${ }^{6}$ Arctic and Antarctic Research Institute, Climate and Environmental Research Laboratory, \\ St. Petersburg, 199397, Russia \\ ${ }^{7}$ Institute of Earth Sciences, Saint Petersburg State University, St. Petersburg, 199034, Russia \\ ${ }^{8}$ Hydrometeorological Research Center of Russian Federation, Moscow, 123242, Russia \\ ${ }^{9}$ Laboratory of Climatology, Institute of Geography, Russian Academy of Science, Moscow, 119017, Russia
}

Correspondence: Ekaterina P. Rets (retska@mail.ru)

Received: 30 September 2018 - Discussion started: 9 October 2018

Revised: 21 June 2019 - Accepted: 15 August 2019 - Published: 30 September 2019

\begin{abstract}
This study presents a dataset on long-term multidisciplinary glaciological, hydrological, and meteorological observations and isotope sampling in a sparsely monitored alpine zone of the North Caucasus in the Djankuat research basin. The Djankuat glacier, which is the largest in the basin, was chosen as representative of the central North Caucasus during the International Hydrological Decade and is one of 30 "reference" glaciers in the world that have annual mass balance series longer than 50 years (Zemp et al., 2009). The dataset features a comprehensive set of observations from 2007 to 2017 and contains yearly

- measurements of snow depth and density;

- measurements of dynamics of snow and ice melting;

- measurements of water runoff, conductivity, turbidity, temperature, $\delta^{18} \mathrm{O}, \delta \mathrm{D}$ at the main gauging station (844 samples in total) with an hourly or sub-daily time step depending on the parameter;

- data on $\delta^{18} \mathrm{O}$ and $\delta^{2} \mathrm{H}$ sampling of liquid precipitation, snow, ice, firn, and groundwater in different parts of the watershed taken regularly during melting season (485 samples in total);

- measurements of precipitation amount, air temperature, relative humidity, shortwave incoming and reflected radiation, longwave downward and upward radiation, atmospheric pressure, and wind speed and direction measured at several automatic weather stations within the basin with $15 \mathrm{~min}$ to $1 \mathrm{~h}$ time steps;
\end{abstract}


- gradient meteorological measurements to estimate turbulent fluxes of heat and moisture, measuring three components of wind speed at a frequency of $10 \mathrm{~Hz}$ to estimate the impulse of turbulent fluxes of sensible and latent heat over the glacier surface by the eddy covariance method.

Data were collected during the ablation period (June-September). The observations were halted in winter. The dataset is available from PANGAEA (https://doi.org/10.1594/PANGAEA.894807, Rets et al., 2018a) and will be further updated. The dataset can be useful for developing and verifying hydrological, glaciological, and meteorological models for alpine areas, to study the impact of climate change on hydrology of mountain regions using isotopic and hydrochemical approaches in hydrology. As the dataset includes the measurements of hydrometeorological and glaciological variables during the catastrophic proglacial lake outburst in the neighboring Bashkara valley in September 2017, it is a valuable contribution to study lake outbursts.

\section{Introduction}

Mountain areas are highly sensitive to climate change (Dyurgerov, 2003; Weingartner et al., 2007; Auer et al., 2007; Viviroli et al., 2011; Pachauri et al., 2014; Zemp et al., 2015). However, it is widely recognized that there is still a significant lack of observational data on climate, glaciers, and hydrology of mountain areas (Gietl, 1990; Barry, 1992; Singh et al., 1999; Schaefli et al., 2005; Bales et al., 2006). The density of hydrological stations in the world's mountainous regions is from 3 (in Europe) to 100 (in Asia) times lower than that recommended by the World Meteorological Organization (Viviroli et al., 2011; Bobrovitskaya and Kokorev, 2014). The majority of field observations in mountainous catchments are conducted in Scandinavia, the Alps, and the mountains of the US, while vast Asian territories and the Southern Hemisphere are sparsely monitored (Barry, 1992; Dyurgerov, 2003; Meier et al., 2003; Zemp et al., 2009; Viviroli et al., 2011; Immerzeel et al., 2012). The Great Caucasus developed an observational network during the Soviet Union period. But recently, has become a poorly instrumented terrain lacking high-quality glaciological and hydrometeorological data (Barry, 1992; Dyurgerov, 2003; Shahgedanova et al., 2005; Bobrovitskaya and Kokorev, 2014).

The specificity of this dataset is a relatively long measurement period of 10 years (2007-2017) including several high discharge events covered in a multi-site measurement program and the extensive set of measured variables, which is unique for the North Caucasus area.

The Djankuat research basin, $9.1 \mathrm{~km}^{2}$ in area, is located at $43.2^{\circ} \mathrm{N}$ and $42.75^{\circ} \mathrm{E}$ in the alpine zone of the North Caucasus (Russia), between 2600 and $4000 \mathrm{~m}$ (Fig. 1). Djankuat glacier, covering $27 \%$ of its area, was chosen as representative of the central North Caucasus during the International Hydrological Decade (IHD) - research program on water problems launched by UNESCO in 1965 (Boyarsky, 1978). The mass-balance measurements have been regularly carried out on Djankuat glacier since 1967 till now (https://wgms. $\mathrm{ch} /$, last access date: 13 September 2019) based on standard methods (Østrem and Brugman, 1991, Boyarsky, 1978). Detailed hydrological and meteorological measurements were included in the monitoring program of the station during the IHD and came to an end in the late 1970s (Boyarsky, 1978). Comprehensive hydrometeorological observation has been resumed in the Djankuat research basin under the initiative of the collective of the authors since 2007. Hydrometeorological measurements were taken during the ablation season every year since 2007 , and the observational program gradually expanded during 2007-2017 and now goes beyond the standard network hydrological and meteorological observations. The relative cutting of the program in 2011-2012 happened due to a special military regime in the Kabardino-Balkaria republic.

Automatic weather stations (AWSs) are installed in four main locations in the basin (see Fig. 1, Table 1). All the meteorological stations operate only during the ablation season of each year. Two Campbell AWSs are located in the central part of the Djankuat glacier above the ice surface (AWS 1) and the debris of the glacier (AWS 2). AWS 1 operated through the period 2007-2017 (excluding 2011). The second Campbell station, located over the debris (AWS 2), operated for 3 years (2007-2009). Both stations provide measurements of air temperature, relative humidity, downward and upward shortwave radiation, downward and upward longwave radiation, wind speed, wind direction, and atmospheric pressure. A Davis weather station operated at base camp (base camp AWS) through 2007-2009 and 2013-2017. The second Davis (AWS 3) station was placed on the upper part of the Djankuat glacier in 2017. A Davis Gradient mast was placed in the central part of the Djankuat glacier (AWS 1) in 2015 to obtain long-term meteorological data series in the surface layer. Turbulent pulsations of wind and acoustic temperature were measured in 2013, 2014, and 2016 with a three-axis Gill Instruments WindMaster sonic anemometer in the central part of the Djankuat glacier (AWS 1).

Hydrological measurements at the Djankuat gauging station (see Fig. 1 and Table 1) started from measuring runoff with a $1 \mathrm{~h}$ step during 2007-2010 ablation seasons. In 2013 the first test measurements of water conductivity, water salinity, water turbidity, and stable isotopes $\left({ }^{18} \mathrm{O}\right.$ and $\left.{ }^{2} \mathrm{H}\right)$ as well as first samplings of liquid precipitation, snow, ice, and firn in the basin for water conductivity, water salinity, $\delta^{18} \mathrm{O}$, 


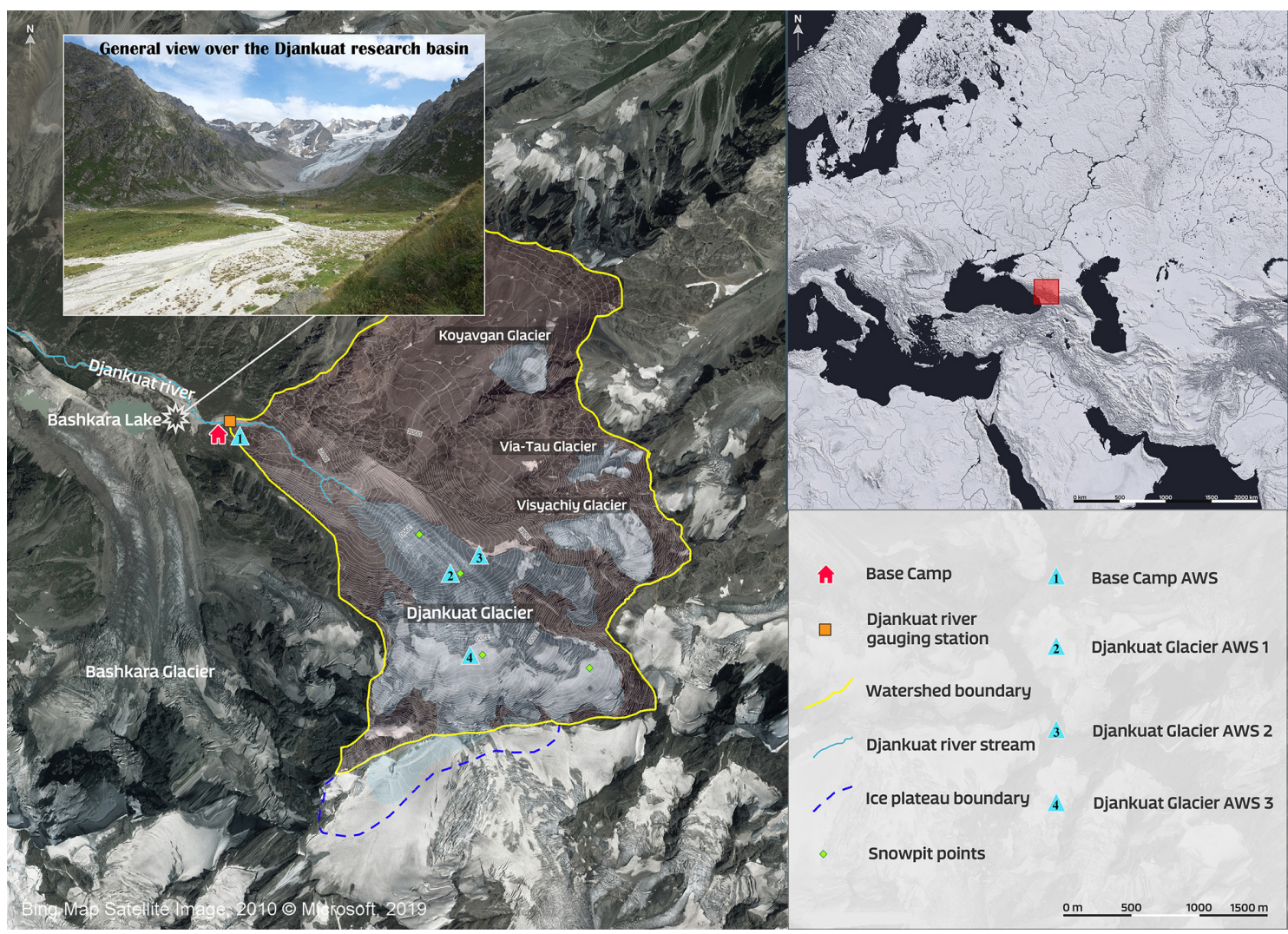

Figure 1. The Djankuat River basin with the depicted location of the base camp, main weather stations, snow pits, and the Djankuat River gauging station.

Table 1. Location of main sites within the Djankuat research basin.

\begin{tabular}{llrrr}
\hline No. Name & $\begin{array}{r}x \text { (longitude), } \\
\text { decimal degrees }\end{array}$ & $\begin{array}{r}y \text { (latitude), } \\
\text { decimal degrees }\end{array}$ & $\begin{array}{r}\text { Elevation, } \\
\mathrm{m}\end{array}$ \\
\hline 1 & Djankuat base camp & 42.735 & 43.208 & 2635 \\
2 & Djankuat base camp AWS & 42.736 & 43.208 & 2640 \\
3 & Djankuat glacier AWS 1 & 42.757 & 43.198 & 3000 \\
4 & Djankuat glacier AWS 2 & 42.759 & 43.2 & 3050 \\
5 & Djankuat glacier AWS 3 & 42.759 & 43.193 & 3200 \\
6 & Djankuat River gauging station & 42.736 & 43.209 & 2630 \\
\hline
\end{tabular}

and $\delta^{2} \mathrm{H}$ were carried out. A total number of 1329 samples on stable isotopes were collected during 2014-2017 in the Djankuat River basin, including 844 samples of the streamflow and 485 samples of snow, ice, firn, groundwater, and liquid precipitation. At the outlet of Djankuat River, turbidity was recorded from 2015 to 2017 , water conductivity from 2014 to 2017, and water temperature from 2015 to 2017.

The dataset was published in a long-term data repository (https://doi.org/10.1594/PANGAEA.894807, Rets et al., 2018a) and will be updated. Outcomes of the research included studies of glacier mass balance (Zemp et al., 2009, 2011, 2015; Rets and Kireeva, 2010; Lambrecht et al., 2011; Popovnin and Pylayeva, 2015), water transport pro- cesses, dangerous hydrological phenomena (Rets et al., 2017; Toropov et al., 2017; Chernomorets et al., 2018), and sediment budget issues (Chalov et al., 2017a).

\section{Study area}

The Greater Caucasus stretches $1300 \mathrm{~km}$ along the borders between Russia and Georgia from the Black Sea to the Caspian Sea. The alpine zone extends above the orographic snowline approximately $2000 \mathrm{~m}$ above sea level. Mount Elbrus has the highest elevation in the Greater Caucasus, reaching $5642 \mathrm{~m}$. The climate is moderate continental to high alpine. The precipitation decreases both southeastwards and 
with a decrease in elevation. The annual precipitation sum varies from 200 to $400 \mathrm{~mm}$ in the eastern plain part and 600$800 \mathrm{~mm}$ in the western plain part to $800-1300 \mathrm{~mm}$ and more in the mountainous part (Rets et al., 2018b). The water supply in the North Caucasus is strongly dominated by runoff formed in the high mountains; a unit of area at an elevation of $3000-4000 \mathrm{~m}$ can be 10 times more productive in terms of water yield than the lowlands. In the most alpine zone of the North Caucasus annual unit discharge varies from 600900 to $1500-1800 \mathrm{~mm}$. In the foothills mean annual runoff unit discharge sharply declines to $150-500 \mathrm{~mm}$ (Rets et al., 2018b).

The Djankuat research basin is situated on the northern slope of the central part of the main Caucasian ridge (see Fig. 1). It is a typical alpine watershed of $9.1 \mathrm{~km}^{2}$ with the elevation range of $2600-4000 \mathrm{~m}$, with steep slopes (more than $20^{\circ}$ on average) and a nival-glacial landscape (see Fig. 1). The overall aspect of the basin is north-northwest. In 2017 glaciers occupied $27 \%$ of the territory of the basin. The main glacier with the same name - Djankuat glacier - is the source of the Djankuat River. It is a valley glacier, with the lowest point of the tongue at approximately $2750 \mathrm{~m}$; the elevation of the bergschrund is at $3600 \mathrm{~m}$. The mean elevation of the glacier is $3210 \mathrm{~m}$, the area is $2.42 \mathrm{~km}^{2}$, and its length is $3.0 \mathrm{~km}$. The maximum measured thickness of the glacier is $105 \mathrm{~m}$, and the average thickness is $31 \mathrm{~m}$ (Lavrentiev et al., 2014). The Djankuat River basin also contains three small glaciers with areas less than $0.5 \mathrm{~km}^{2}$ : Koyavgan, Via-Tau, and Visyachiy. These glaciers contribute runoff to the Djankuat River upstream, where the main gauging station is located (see Fig. 1). The Djankuat River is a source of the Adul-Su River - a tributary of the Baksan River, which drains into the Caspian Sea via the Terek River.

The uppermost gauging station on the Baksan River was situated $12 \mathrm{~km}$ from its source in Usengi. The river basin area at the gauging station is $180 \mathrm{~km}^{2}$. The mean annual discharge of the Baksan River at Usengi gauging station is $9.9 \mathrm{~m}^{3} \mathrm{~s}^{-1}$, which amounts to mean annual runoff of $1700 \mathrm{~mm}$ (Rets and Kireeva, 2010). The water-abundant period of the Baksan River in the upstream is prolonged and steady; it extends from May to September-October. The river hydrograph has a sawtooth form due to the rain flash floods overlapping the general snow and ice melting wave. The maximum water levels are usually recorded in July. A stable winter low-flow period with minimum monthly discharge of $2.4 \mathrm{~m}^{3} \mathrm{~s}^{-1}$ is observed from February to March (Rets and Kireeva, 2010). Annual river runoff in the mountainous part of the North Caucasus shows a slightly positive trend during 1940-2010. In the most elevated areas, the long-term mean value of annual runoff remains stable (Rets et al., 2018b).

A distinct seasonality in temperature characterizes the climate of the Djankuat research basin. The mean monthly air temperatures at the Terskol, the all-year meteorological station closest to the research basin, situated $16 \mathrm{~km}$ northwest of the study area at an altitude of $2146 \mathrm{~m}$, drop below zero during November-December. The warmest months are July and August with mean monthly temperatures above $12^{\circ} \mathrm{C}$ (Fig. 2). Monthly precipitation totals are $40 \%-50 \%$ higher in the warm period of the year (May-September) than during winter (Fig. 2). The annual precipitation at the Terskol weather station varied from 590 to $1330 \mathrm{~mm}$ with a mean annual value of $950 \mathrm{~mm}$. Daily precipitation maxima occur from July to September in response to convective activity triggered by a combination of intense insolation and depressions developing on the polar front and enhanced by the orographic uplift (Shahgedanova, 2002).

The observations in the Djankuat research basin, included in the presented dataset, were carried out under the conditions of slightly warmer summer periods in comparison to the long-term average, and substantially higher amounts of precipitation, especially during the spring period - from March to May (Fig. 2). The strong influence of spring snowfalls during the observation period is also reflected in the results of Djankuat glacier snow sampling for stable isotopes (Rets et al., 2017). The outlined tendency is likely to be the consequence of climate change in the region.

According to the majority of studies (Alekseev et al., 2014; Toropov et al., 2018a; Rets and Kireeva, 2010), a statistically significant positive trend in air temperature amounting to $0.7-1^{\circ} \mathrm{C}$ per decade is observed during the summer period in the North Caucasus. A slight positive trend in the mean temperature in the ablation period (May to September), $0.3{ }^{\circ} \mathrm{C}$ per decade, has been observed at the Terskol meteorological station since the end of the 1970s. In the winter period, the observed tendencies in air temperature in the North Caucasus are very inhomogeneous: Alekseev et al. (2014) report a statistically insignificant positive trend. In the study by Rets and Kireeva (2010), a decrease in air temperature of the winter period was revealed in the mountainous part of the North Caucasus. According to the Terskol meteorological station, the value of mean air temperature during the accumulation season (October to April) remains stable in the study area.

According to different studies, a positive trend in annual precipitation sum of $5 \%$ per decade is reported by Alekseev et al. (2014), and no statically significant trend is reported for most of the North Caucasus by Toropov et al. (2018a). An increase in annual precipitation sum was revealed for the majority of mountainous stations and several foothills located in the central part of the Northern Caucasus (Rets and Kireeva, 2010). At the Terskol meteorological station, the amount of precipitation has been constantly rising during the whole observational periods. The increase in annual sum of $3.5 \%$ per decade is different for the ablation $(2.1 \%$ per decade) and accumulation (5\% per decade) period. The most intensive rise in precipitation is observed in spring $(8.6 \%$ per decade in March, $7 \%$ per decade in April) and autumn (10.3\% per decade in October). This result is consistent with the result reported by Alekseev et al. (2014) for the whole territory of the North Caucasus. 


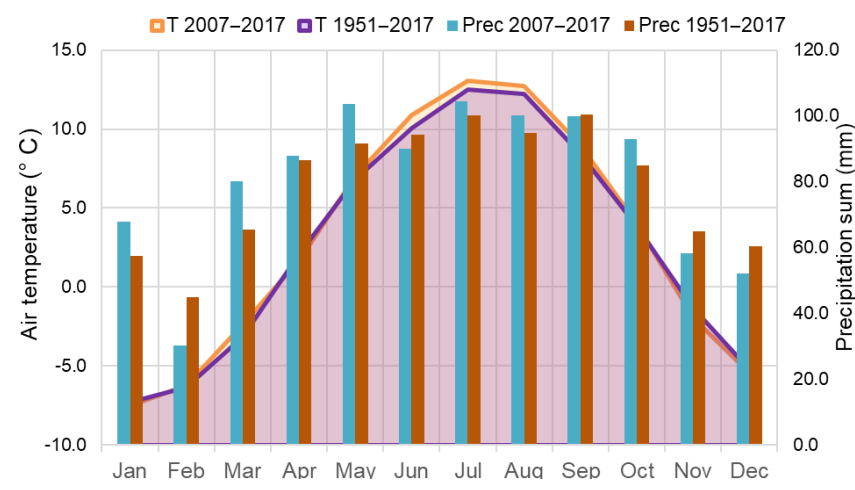

Figure 2. Monthly distribution of air temperature and precipitation for the period of the dataset (2007-2017) compared to the long-term period (1951-2017) at the all-year weather station Terskol nearest to the Djankuat research basin $(2146 \mathrm{~m})$.

An intensive deglaciation is observed in the North Caucasus (Shahgedanova et al., 2014; Zemp et al., 2015). The area of glaciers in the North Caucasus dropped by $12.6 \%$ during 1970-2000 (Volodicheva and Voitkovskiy, 2004) and by $4.7 \%$ between 2000 and 2010-2012 (Shahgedanova et al., 2014), amounting to approximately $17 \%$ in total during 1970-2012. The glacier terminus retreat increased from the $1987-2000 / 2001$ period to the $2000 / 2001-2010$ period by a factor of 2.5-3.8 (Shahgedanova et al., 2014). Glacier retreat and the increase in supraglacial debris cover is also accompanied by the emergence and growth of proglacial lakes and related increase in proglacial lake outburst floods (Stokes et al., 2007). On 1 September 2017, an outburst of Bashkara lake upstream neighboring the Djankuat basin valley gave rise to catastrophic mudflow that led to major destruction and human casualties (Chernomorets et al., 2018).

\section{Methods and results}

\subsection{Hydrological measurements}

\subsubsection{Discharge measurements}

Water discharge of the Djankuat River at the gauging station (see Fig. 1, see Table 1) was calculated from the water level using a rating curve $Q=f(H)$ (Table 2a). The water level was recorded with a $10 \mathrm{~min}$ to hourly time step (depending on the year of observation) by means of an automatic water level logger with a pressure sensor (ADU-02 during 20072013, Solinst level logger in 2014-2017). The level logger was placed with a ripple shield in an artificial bay constructed at the river bank (Fig. 3b). Additionally, manual water level measurements were taken six to seven times a day. Rating curves were drawn for each month on a yearly basis. Dilution using $\mathrm{NaCl}$ as a tracer was performed for discharge metering as turbulent flow conditions make it impossible to apply the current meter (Dobriyal et al., 2017).
As there was a possibility of erroneous results due to the loss or incomplete mixing of the tracer arising from the difference in velocity in the upper and lower layers of the stream (Dobriyal et al., 2017), every discharge measurement was repeated twice. The value was accepted if the difference between the two simultaneous measurements did not exceed $10 \%$. For most of the discharge measurements at the Djankuat gauging station, this difference was less than $5 \%$. A total of 50-80 water discharges were measured every ablation season to draw a rating curve.

Water discharge at the Djankuat gauging station was observed within a range of 1 to $2 \mathrm{~m}^{3} \mathrm{~s}^{-1}$ in 2007-2017 (Fig. 4a), the mean value of water discharge was $1.39 \mathrm{~m}^{3} \mathrm{~s}^{-1}$ (Table 3). During high discharge events (1\% probability) streamflow rates ranged from 3.5 to $8.46 \mathrm{~m}^{3} \mathrm{~s}^{-1}$. The maximum discharge, $8.46 \mathrm{~m}^{3} \mathrm{~s}^{-1}$, was observed on 1 July 2015 at 09:00 UTC +3 (for all times throughout) due to heavy flooding caused by $227 \mathrm{~mm}$ of precipitation over $7 \mathrm{~d}$ superimposed on intensive snow and ice melting in the river basin.

The inter-annual fluctuations of the Djankuat River runoff can be quite substantial (Fig. 5). The Djankuat River was the most abundant in water during 2015-2016 and the least in 2013. Mean water discharge for June to September in 2015 $\left(1.88 \mathrm{~m}^{3} \mathrm{~s}^{-1}\right)$ was twice as large as in $2013\left(0.97 \mathrm{~m}^{3} \mathrm{~s}^{-1}\right)$. The Djankuat River is most abundant in water in July (Fig. 5). Mean monthly discharge in this month is $1.3-2.8 \mathrm{~m}^{3} \mathrm{~s}^{-1}$. June and August are comparable in terms of mean discharge, 0.93 to 1.8 and 1.0 to $2.0 \mathrm{~m}^{3} \mathrm{~s}^{-1}$, respectively. In September, runoff due to ice and firn melting decreases with the decrease in the incoming solar radiation and the seasonal meltwater is gradually drained from the Djankuat River basin. Mean monthly discharge is $0.6-1 \mathrm{~m}^{3} \mathrm{~s}^{-1}$. At the end of September, the ablation period ends with the first stable fresh snow cover on the glacier.

The Djankuat River hydrograph has a sawtooth shape with a pronounced daily maximum and minimum typical for glacial rivers (Fig. 6). A diurnal fluctuation of discharge is large and can be compared with the overall seasonal fluctuation: up to $1.5-2 \mathrm{~m}^{3} \mathrm{~s}^{-1}$ on a day without rain. The rise of rain flash-flooding can be very intensive: more than $1 \mathrm{~m}^{3} \mathrm{~s}^{-1}$ per hour.

\subsubsection{Electrical conductivity and salinity}

Water salinity was calculated from the conductivity measurements (Table 2a), using a dependency salinity $=f$ (cond). The dependency was drawn in 2013 using the data on simultaneous measurement of electrical conductivity and complete chemical analysis in 19 samples with conductivity from 4.2 to $87.5 \mu \mathrm{S} \mathrm{cm}^{-1}$. The Djankuat River water has a low salinity. The value of electrical conductivity stayed in the range of 55$85 \mu \mathrm{S} \mathrm{cm}^{-1}$ for $90 \%$ of the time (Table 3, Fig. $4 \mathrm{~d}$ ). The electrical conductivity value strongly depends on the percentage of snow and ice meltwater in the total river runoff. During long periods without rain with intensive melting, the water 
Table 2. (a) Hydrological characteristics measured in the Djankuat research basin and included in the database. (b) Glaciological characteristics measured in the Djankuat research basin and included in the database. (c) Meteorological characteristics measured in the Djankuat research basin and included in the database.

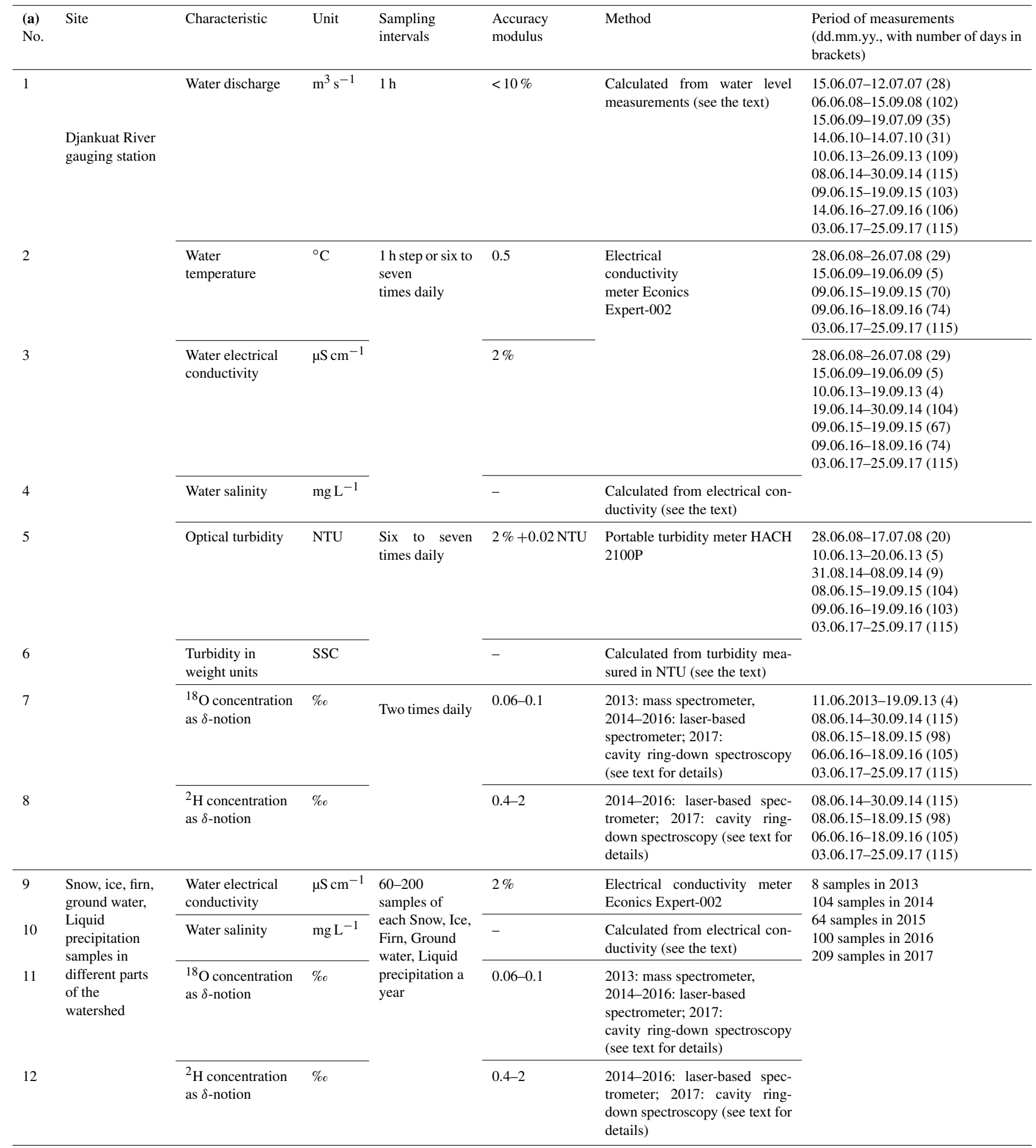


Table 2. Continued.

\begin{tabular}{|c|c|c|c|c|c|c|c|}
\hline $\begin{array}{l}\text { (b) } \\
\text { No. }\end{array}$ & Site & Characteristic & Unit & $\begin{array}{l}\text { Sampling } \\
\text { intervals }\end{array}$ & $\begin{array}{l}\text { Accuracy } \\
\text { modulus }\end{array}$ & Method & Period of measurements \\
\hline 1 & $\begin{array}{l}\text { At } 250-300 \\
\text { evenly dis- } \\
\text { tributed points } \\
\text { on the Djankuat } \\
\text { glacier surface }\end{array}$ & Snow thickness & $\mathrm{cm}$ & $\begin{array}{l}\text { One time } \\
\text { yearly }\end{array}$ & 5 & Probe poles & \begin{tabular}{|l|}
$12.06 .07-30.06 .07$ \\
$12.06 .08-07.07 .08$ \\
$08.06 .09-07.07 .09$ \\
$19.05 .10-10.07 .10$ \\
$04.06 .12-06.07 .12$ \\
$05.06 .13-01.07 .13$ \\
$04.06 .14-04.07 .14$ \\
$04.06 .15-12.07 .15$ \\
$26.05 .16-15.07 .16$ \\
$04.06 .17-11.07 .17$
\end{tabular} \\
\hline 2 & $\begin{array}{l}\text { At } 35-45 \\
\text { evenly dis- } \\
\text { tributed points } \\
\text { on the Djankuat } \\
\text { glacier surface }\end{array}$ & Ablation & $\begin{array}{l}\mathrm{mm} \text { of } \\
\text { water } \\
\text { equiva- } \\
\text { lent }\end{array}$ & $1-30 d$ & 2 & Ablation stakes & \begin{tabular}{|l|}
$12.06 .07-10.09 .07$ \\
$16.05 .08-07.10 .08$ \\
$08.06 .09-25.09 .09$ \\
$18.06 .10-02.10 .10$ \\
$03.06 .12-04.10 .12$ \\
$05.06 .13-21.09 .13$ \\
$04.06 .14-30.09 .14$ \\
$04.06 .15-20.09 .15$ \\
$26.05 .16-20.09 .16$ \\
$03.06 .17-24.09 .17$
\end{tabular} \\
\hline 3 & $\begin{array}{l}\text { Stakes } 9,18,49, \\
65 / 68\end{array}$ & Snow density & $\mathrm{g} \mathrm{cm}^{-3}$ & $\begin{array}{l}\text { One to five } \\
\text { times in } \\
\text { each snow pit } \\
\text { yearly }\end{array}$ & 0.01 & Snow sampling cylinder & $\begin{array}{l}19.05 .07-13.06 .07 \\
16.05 .08-14.07 .08 \\
03.06 .09-01.09 .09 \\
21.05 .10-06.07 .10 \\
04.06 .12-06.07 .12 \\
06.06 .13-22.06 .13 \\
05.06 .14-02.09 .14 \\
05.06 .15-04.09 .15 \\
29.05 .16-07.10 .16 \\
06.06 .17-10.08 .17\end{array}$ \\
\hline $\begin{array}{l}\text { (c) } \\
\text { No. }\end{array}$ & Site & Characteristic & Unit & $\begin{array}{l}\text { Sampling } \\
\text { intervals }\end{array}$ & $\begin{array}{l}\text { Accuracy } \\
\text { modulus }\end{array}$ & Method & $\begin{array}{l}\text { Period of measurements (with } \\
\text { number of days in brackets) }\end{array}$ \\
\hline 1 & \multirow{10}{*}{$\begin{array}{l}\text { Djankuat } \\
\text { glacier AWS } 1\end{array}$} & $\begin{array}{l}\text { Air temperature at } \\
2 \mathrm{~m}\end{array}$ & ${ }^{\circ} \mathrm{C}$ & & 0.2 & $\begin{array}{l}\text { Campbell AWS }- \text { Vaisala } \\
\text { MT300 sensor }\end{array}$ & \multirow{10}{*}{$\begin{array}{l}15.06 .07-30.09 .07(107) \\
17.06 .08-30.09 .08(105) \\
01.07 .09-30.09 .09(91) \\
09.07 .10-29.09 .10(82) \\
10.07 .12-05.08 .12(26) \\
07.07 .13-09.09 .13(64) \\
19.06 .14-30.09 .14(103) \\
07.07 .15-04.09 .15(59) \\
20.06 .16-05.09 .16(77) \\
19.06 .17-02.09 .17(75)\end{array}$} \\
\hline 2 & & $\begin{array}{l}\text { Relative humidity } \\
\text { at } 2 \mathrm{~m}\end{array}$ & $\%$ & & 5 & & \\
\hline 3 & & Wind speed at $2 \mathrm{~m}$ & $\mathrm{~ms}^{-1}$ & $15 \mathrm{~min}$ & $(0.5+0.05 \mathrm{~W})$ & Campbell AWS - wind sensor & \\
\hline 4 & & $\begin{array}{l}\text { Wind diection at } \\
2 \mathrm{~m}\end{array}$ & $\circ$ & & 10 & & \\
\hline 5 & & $\begin{array}{l}\text { Atmospheric } \\
\text { pressue }\end{array}$ & $\mathrm{hPa}$ & & 1 & $\begin{array}{l}\text { Campbell AWS - Vaisala pres- } \\
\text { sure sensor }\end{array}$ & \\
\hline 6 & & $\begin{array}{l}\text { Incoming short- } \\
\text { wave radiation }\end{array}$ & $\mathrm{Wm}^{-2}$ & & 15 & $\begin{array}{l}\text { Campbell AWS - Kipp \& Zo- } \\
\text { nen radiometers }\end{array}$ & \\
\hline 7 & & $\begin{array}{l}\text { Reflected short- } \\
\text { wave radiation }\end{array}$ & $\mathrm{Wm} \mathrm{m}^{-3}$ & & & & \\
\hline 8 & & $\begin{array}{l}\text { Downward long- } \\
\text { wave radiation }\end{array}$ & $\mathrm{W} \mathrm{m}^{-4}$ & & & & \\
\hline 9 & & $\begin{array}{l}\text { Upward longwave } \\
\text { radiation }\end{array}$ & $\mathrm{W} \mathrm{m}^{-2}$ & & & & \\
\hline 10 & & $\begin{array}{l}\text { Sensor-surface } \\
\text { distance }\end{array}$ & $\mathrm{m}$ & & 0.01 & $\begin{array}{l}\text { Campbell AWS - Sonic Ranger } \\
\text { sensor }\end{array}$ & \\
\hline
\end{tabular}


Table 2. Continued.

\begin{tabular}{|c|c|c|c|c|c|c|c|}
\hline $\begin{array}{l}\text { (c) } \\
\text { No. }\end{array}$ & Site & Characteristic & Unit & $\begin{array}{l}\text { Sampling } \\
\text { intervals }\end{array}$ & $\begin{array}{l}\text { Accuracy } \\
\text { modulus }\end{array}$ & Method & $\begin{array}{l}\text { Period of measurements (with } \\
\text { number of days in brackets) }\end{array}$ \\
\hline 11 & & $\begin{array}{l}\text { Air temperature at } \\
0.25,0.5,1 \text { and } 2 \mathrm{~m}\end{array}$ & ${ }^{\circ} \mathrm{C}$ & & \pm 0.4 & \multirow{5}{*}{ DAVIS AWS } & \multirow{5}{*}{$05.07 .15-15.08 .15(41)$} \\
\hline 12 & & $\begin{array}{l}\text { Relative humidity at } \\
0.25,0.5,1 \text { and } 2 \mathrm{~m}\end{array}$ & $\%$ & & \pm 10 & & \\
\hline 13 & & $\begin{array}{l}\text { Mean wind } \\
\text { speed at } 0.25,0.5,1 \\
\text { and } 2 \mathrm{~m}\end{array}$ & $\mathrm{~ms}^{-1}$ & & $\pm 0.5-2$ & & \\
\hline 14 & & $\begin{array}{l}\text { Maximum wind } \\
\text { speed at } 0.25,0.5,1 \\
\text { and } 2 \mathrm{~m}\end{array}$ & $\mathrm{~ms}^{-1}$ & & & & \\
\hline 15 & & $\begin{array}{l}\text { Wind direction at } \\
0.25,0.5,1 \text { and } 2 \mathrm{~m}\end{array}$ & rhumb & & - & & \\
\hline 16 & & $\begin{array}{l}\text { Three components } \\
\text { of wind speed }\end{array}$ & $\mathrm{ms}^{-1}$ & \multirow[t]{2}{*}{$10 \mathrm{~Hz}$} & $0.05-0.1$ & \multirow[t]{2}{*}{ Sonic anemometer GILL } & \multirow[t]{2}{*}{$\begin{array}{l}12.07 .13-03.08 .13(22) \\
09.08 .13-16.08 .13(7) \\
26.08 .13-06.09 .13(42) \\
30.06 .14-30.07 .14(30) \\
17.06 .16-01.08 .16(45)\end{array}$} \\
\hline 17 & & $\begin{array}{l}T-\text { acoustic temper- } \\
\text { ature }\end{array}$ & ${ }^{\circ} \mathrm{C}$ & & & & \\
\hline 18 & \multirow{10}{*}{$\begin{array}{l}\text { Djankuat } \\
\text { glacier AWS } 2\end{array}$} & $\begin{array}{l}\text { Air temperature at } \\
2 \mathrm{~m}\end{array}$ & ${ }^{\circ} \mathrm{C}$ & \multirow{10}{*}{$15 \mathrm{~min}$} & 0.2 & $\begin{array}{l}\text { Campbell AWS - } \\
\text { Vaisala MT300 sensor }\end{array}$ & \multirow{10}{*}{$\begin{array}{l}17.06 .07-07.09 .07(82) \\
02.07 .08-26.09 .08(86) \\
23.06 .09-02.10 .09(101)\end{array}$} \\
\hline 19 & & $\begin{array}{l}\text { Relative humidity at } \\
2 \mathrm{~m}\end{array}$ & $\%$ & & 5 & & \\
\hline 20 & & Wind speed at $2 \mathrm{~m}$ & $\mathrm{~ms}^{-1}$ & & $(0.5+0.05 \mathrm{~W})$ & Campbell AWS - & \\
\hline 21 & & $\begin{array}{l}\text { Wind direction at } \\
2 \mathrm{~m}\end{array}$ & $\circ$ & & 10 & & \\
\hline 22 & & $\begin{array}{l}\text { Atmospheric } \\
\text { pressure }\end{array}$ & $\mathrm{hPa}$ & & 1 & $\begin{array}{l}\text { Campbell AWS - Vaisala pres- } \\
\text { sure sensor }\end{array}$ & \\
\hline 23 & & $\begin{array}{l}\text { Incoming shortwave } \\
\text { radiation }\end{array}$ & $\mathrm{W} \mathrm{m}^{-2}$ & & 15 & \multirow{4}{*}{$\begin{array}{l}\text { Campbell AWS - } \\
\text { KEEP \& ZONNEN } \\
\text { radiometers }\end{array}$} & \\
\hline 24 & & $\begin{array}{l}\text { Reflected shortwave } \\
\text { radiation }\end{array}$ & $\mathrm{W} \mathrm{m}^{-3}$ & & & & \\
\hline 25 & & $\begin{array}{l}\text { Downward } \\
\text { longwave radiation }\end{array}$ & $\mathrm{W} \mathrm{m}^{-4}$ & & & & \\
\hline 26 & & $\begin{array}{l}\text { Upward longwave } \\
\text { radiation }\end{array}$ & $\mathrm{W} \mathrm{m}^{-2}$ & & & & \\
\hline 27 & & $\begin{array}{l}\text { Sensor-surface } \\
\text { distance }\end{array}$ & $\mathrm{m}$ & & $0.04-0.06$ & $\begin{array}{l}\text { Campbell AWS - Sonic Ranger } \\
\text { sensor }\end{array}$ & \\
\hline 28 & \multirow{4}{*}{$\begin{array}{l}\text { Djankuat } \\
\text { glacier AWS } 3\end{array}$} & Air temperature & ${ }^{\circ} \mathrm{C}$ & \multirow{4}{*}{$15 \mathrm{~min}$} & 0.4 & \multirow{4}{*}{ DAVIS AWS } & \multirow{4}{*}{$19.06 .17-21.08 .17(63)$} \\
\hline 29 & & Relative humidity & $\%$ & & 10 & & \\
\hline 30 & & Wind speed & $\mathrm{ms}^{-1}$ & & $(0.6+0.06 \mathrm{~W})$ & & \\
\hline 31 & & Wind direction & $\circ$ & & 15 & & \\
\hline 32 & \multirow{6}{*}{$\begin{array}{l}\text { Base camp } \\
\text { AWS }\end{array}$} & Air temperature & ${ }^{\circ} \mathrm{C}$ & \multirow{5}{*}{$15 \mathrm{~min}$} & 0.4 & \multirow{5}{*}{ DAVIS AWS } & \multirow{6}{*}{$\begin{array}{l}17.06 .07-14.09 .07(90) \\
12.06 .08-20.09 .08(101) \\
26.06 .09-04.10 .09(101) \\
08.06 .13-07.09 .13(92) \\
09.06 .14-01.10 .14(115) \\
09.06 .15-18.09 .15(102) \\
09.06 .16-01.10 .16(115) \\
06.06 .17-25.06 .17(112)\end{array}$} \\
\hline 33 & & Relative humidity & $\%$ & & 10 & & \\
\hline 34 & & Wind speed & $\mathrm{ms}^{-1}$ & & $(0.6+0.06 \mathrm{~W})$ & & \\
\hline 35 & & Wind diection & $\circ$ & & 15 & & \\
\hline 36 & & $\begin{array}{l}\text { Atmospheric } \\
\text { pressue }\end{array}$ & $\mathrm{hPa}$ & & 1 & & \\
\hline 37 & & Liquid precipitation & $\mathrm{mm}$ & $24 \mathrm{~h}$ & $5 \%$ of total & Rain gauge & \\
\hline
\end{tabular}




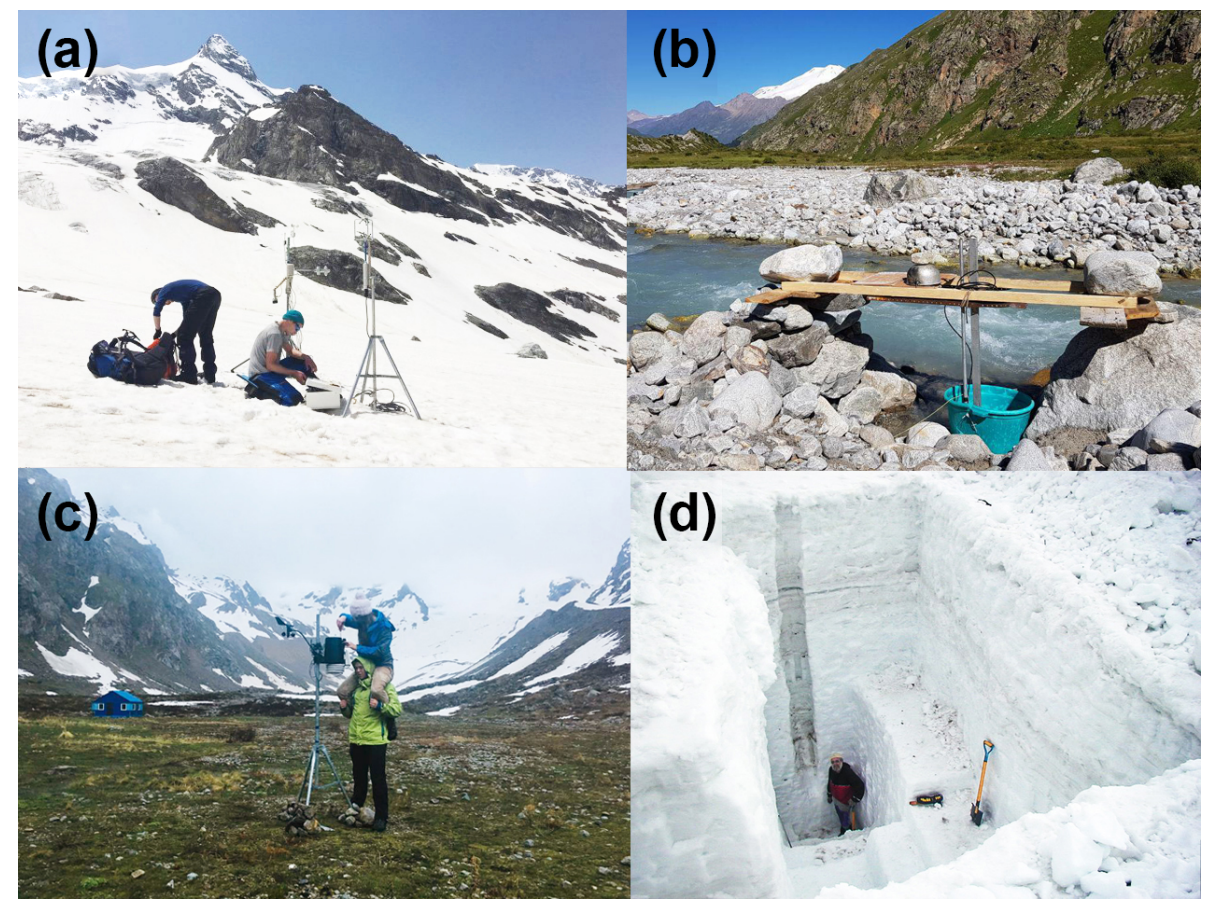

Figure 3. (a) Campbell meteorological complex with a set of Kipp \& Zonen radiometers and a Gill three-component acoustic anemometer at the Djankuat glacier AWS 1 location; (b) the construction of the Djankuat gauging station; (c) Djankuat base camp AWS - Davis meteorological station. (d) Snow density measurement in a snow pit at the stake 49.
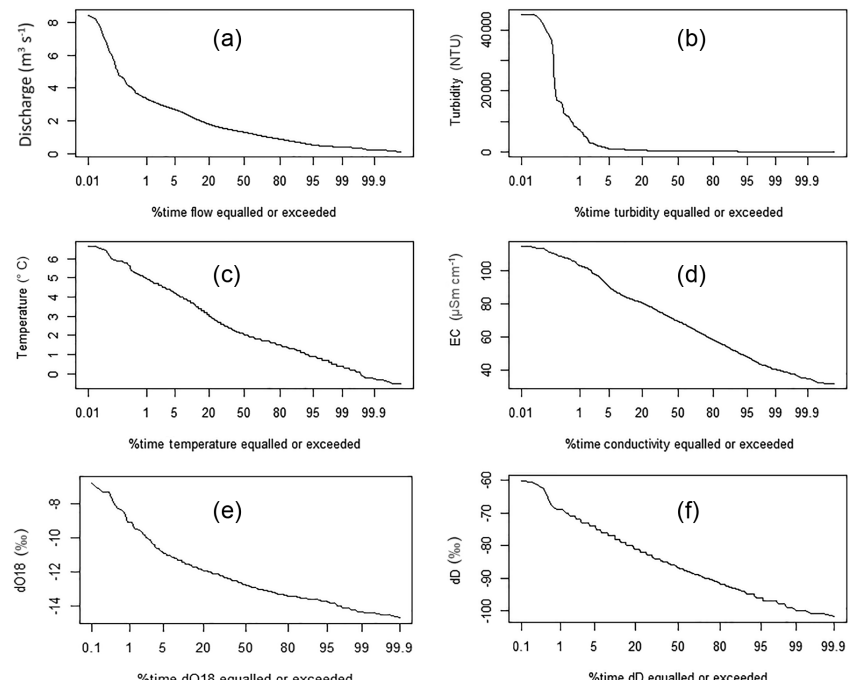

Figure 4. Duration curves of the parameters measured during 2007-2017 at the Djankuat gauging station: (a) water discharge, (b) optical turbidity, (c) water temperature, (d) water electrical conductivity, (e) $\delta^{18} \mathrm{O}$, (f) $\delta \mathrm{D}$.

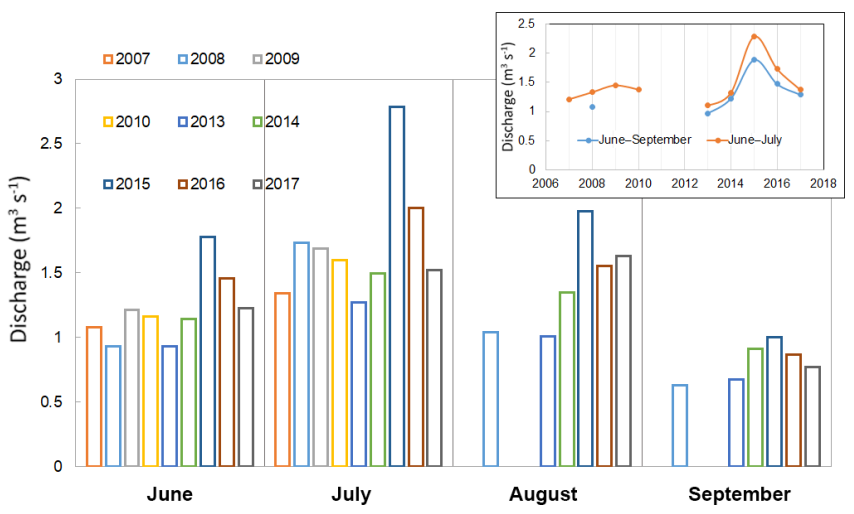

Figure 5. Fluctuations of mean monthly and mean seasonal runoff of Djankuat River during the whole period of observation (20072017).

of the Djankuat River can be diluted up to $40-50 \mu \mathrm{S} \mathrm{cm}^{-1}$ during daytime. For the daily minimums of water discharge in the early morning, the electrical conductivity rises by 10 $30 \mu \mathrm{S} \mathrm{cm}^{-1}$. At the end of the ablation season, when melting is strongly reduced, the electrical conductivity of the Djankuat River reaches values of 110 to $115 \mu \mathrm{S} \mathrm{cm}^{-1}$ during night-morning hours, which is close to the value of electrical conductivity of groundwater in the basin. 
Table 3. Mean value and range of some of the parameters measured in the Djankuat research basin.

\begin{tabular}{|c|c|c|c|c|c|c|}
\hline Parameter & Site & Unit & Number of values & Mean & Max & Min \\
\hline \multirow{4}{*}{$\begin{array}{l}\text { Water discharge } \\
\text { Water temperature } \\
\text { Optical turbidity } \\
\text { Weight turbidity }\end{array}$} & \multirow{4}{*}{ Djankuat River gauging station } & $\mathrm{m}^{3} \mathrm{~s}^{-1}$ & 16971 & 1.39 & 8.46 & 0.11 \\
\hline & & ${ }^{\circ} \mathrm{C}$ & 3259 & 2.26 & 6.63 & 0.1 \\
\hline & & NTU & 1991 & 468 & 45060 & 6.9 \\
\hline & & $\mathrm{gL}^{-1}$ & 1991 & 368 & 37164 & 28 \\
\hline \multirow{5}{*}{$\begin{array}{l}\text { Water electrical } \\
\text { conductivity }\end{array}$} & Djankuat River gauging station & \multirow{5}{*}{$\mu \mathrm{S} \mathrm{cm}^{-1}$} & 3464 & 56.76 & 115 & 31.7 \\
\hline & Snow samples & & 218 & 9.3 & 37.2 & 0.3 \\
\hline & Ice samples & & 116 & 14.8 & 63.7 & 0.7 \\
\hline & Firn samples & & 22 & 13.7 & 23 & 4.1 \\
\hline & Groundwater samples & & 16 & 109 & 115 & 104 \\
\hline \multirow{6}{*}{$\delta^{18} \mathrm{O}$} & Djankuat River gauging station & \multirow{6}{*}{$\%$} & 844 & -12.6 & -6.7 & -14.7 \\
\hline & Snow samples & & 218 & -12.2 & -5 & -28.3 \\
\hline & Ice samples & & 116 & -14.3 & -9.8 & -22 \\
\hline & Firn samples & & 22 & -11.3 & -8.1 & -16.2 \\
\hline & Liquid precipitation samples & & 113 & -4.9 & 5.6 & -16.9 \\
\hline & Groundwater samples & & 16 & -13.3 & -12.3 & -13.6 \\
\hline \multirow{6}{*}{$\delta \mathrm{D}$} & Djankuat River gauging station & \multirow{6}{*}{$\%$} & 842 & -86.2 & -60 & -102 \\
\hline & Snow samples & & 218 & -85.5 & -28 & -216 \\
\hline & Ice samples & & 116 & -99.3 & -64 & -160 \\
\hline & Firn samples & & 22 & -77.1 & -53 & -116 \\
\hline & Liquid precipitation samples & & 113 & -26.2 & 32 & -124 \\
\hline & Groundwater samples & & 16 & -91.1 & -77 & -93.5 \\
\hline \multirow{3}{*}{$\begin{array}{l}\text { Snow thickness } \\
\text { Ablation } \\
\text { Mean snow density for the } \\
\text { snowpack }\end{array}$} & \multirow{4}{*}{ Djankuat glacier } & $\mathrm{cm}$ & 2932 & 360 & 1155 & 7 \\
\hline & & mm w.e. $\mathrm{d}^{-1}$ & 5045 & 47 & 387 & 0 \\
\hline & & $\mathrm{g} \mathrm{cm}^{-3}$ & 66 & 0.57 & 0.64 & 0.46 \\
\hline $\begin{array}{l}\text { Snow density by layers of } \\
\text { the snowpack }\end{array}$ & & $\mathrm{g} \mathrm{cm}^{-3}$ & 434 & 0.57 & 0.92 & 0.23 \\
\hline \multirow{3}{*}{$\begin{array}{l}\text { Air temperature } \\
\text { at } 2 \mathrm{~m} \_ \text {hourly }\end{array}$} & Base camp AWS & \multirow{3}{*}{${ }^{\circ} \mathrm{C}$} & 5340 & 10.2 & 24.2 & -1.10 \\
\hline & Djankuat glacier AWS 1 & & 17551 & 6.60 & 17.6 & -8.97 \\
\hline & Djankuat glacier AWS 2 & & 4901 & 6.37 & 15.8 & -4.22 \\
\hline \multirow{3}{*}{$\begin{array}{l}\text { Relative humidity } \\
\text { at } 2 \mathrm{~m} \_ \text {hourly }\end{array}$} & Base camp AWS & \multirow{3}{*}{$\%$} & 5340 & 65.0 & 95.0 & 12.5 \\
\hline & Djankuat glacier AWS 1 & & 17526 & 68.4 & 100.0 & 13.9 \\
\hline & Djankuat glacier AWS 2 & & 4901 & 68.0 & 100.0 & 10.5 \\
\hline \multirow{3}{*}{$\begin{array}{l}\text { Wind speed at } \\
2 \mathrm{~m} \_ \text {hourly }\end{array}$} & Base camp AWS & \multirow{3}{*}{$\mathrm{ms}^{-1}$} & 5095 & 1.93 & 10.8 & 0.10 \\
\hline & Djankuat glacier AWS 1 & & 17469 & 3.95 & 16.6 & 0.00 \\
\hline & Djankuat glacier AWS 2 & & 6095 & 5.50 & 21.1 & 0.01 \\
\hline \multirow{2}{*}{$\begin{array}{l}\text { Incoming shortwave } \\
\text { radiation_hourly }\end{array}$} & Djankuat glacier AWS 1 & \multirow{2}{*}{$\mathrm{W} \mathrm{m}^{-2}$} & 13728 & 273.8 & 1150.0 & 0.0 \\
\hline & Djankuat glacier AWS 2 & & 3706 & 330.2 & 1160.0 & 0.0 \\
\hline \multirow{2}{*}{$\begin{array}{l}\text { Downward longwave } \\
\text { radiation_hourly }\end{array}$} & Djankuat glacier AWS 1 & \multirow{2}{*}{$\mathrm{W} \mathrm{m}^{-2}$} & 16920 & 295.4 & 407.9 & 161.5 \\
\hline & Djankuat glacier AWS 2 & & 6466 & 286.6 & 382.5 & 156.0 \\
\hline \multirow{2}{*}{$\begin{array}{l}\text { Upward longwave } \\
\text { radiation_hourly }\end{array}$} & Djankuat glacier AWS 1 & \multirow{2}{*}{$\mathrm{W} \mathrm{m}^{-2}$} & 17548 & 313.7 & 408.9 & 171.7 \\
\hline & Djankuat glacier AWS 2 & & 6466 & 353.1 & 494.9 & 156.8 \\
\hline $\begin{array}{l}\text { Liquid precipitation daily } \\
\text { sum }\end{array}$ & Base camp AWS & $\mathrm{mm} \mathrm{d}^{-1}$ & 232 & 11.2 & 97.2 & 0.1 \\
\hline
\end{tabular}

\subsubsection{Water temperature}

Water temperature has a close to uniform distribution on the duration curve and is mostly within the range of $1.2-4.5^{\circ} \mathrm{C}$ (Fig. 4c). Water temperature of the Djankuat River has a diurnal variation up to $4{ }^{\circ} \mathrm{C}$. Diurnal maximum of temperature is usually observed at daytime before the beginning of an intensive rise of diurnal wave of meltwater flow. Mean daily values of water temperature generally rise through the ablation season (Fig. 6). The maximum value $\left(6.63^{\circ} \mathrm{C}\right)$ was registered on 18 September 2016 at midday. The minimum values $\left(0.1^{\circ} \mathrm{C}\right)$ were observed during the night in the beginning of the ablation period (Fig. 6). 


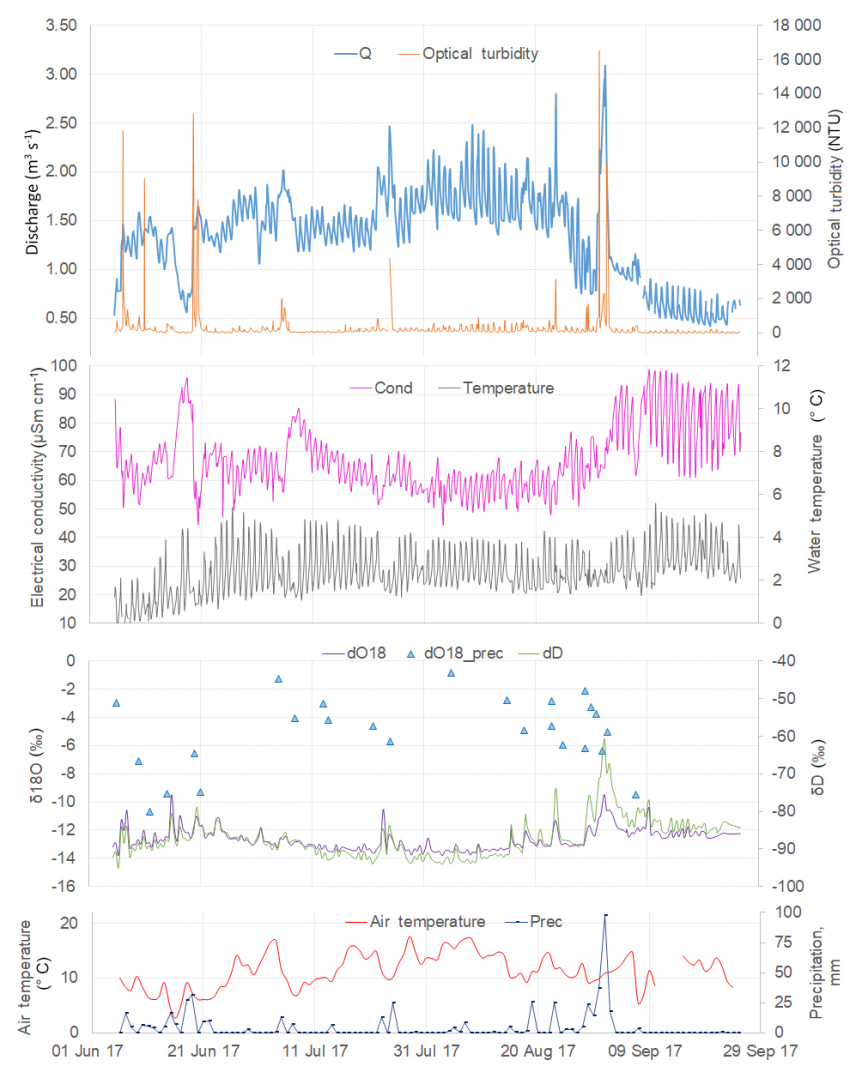

Figure 6. Example of the fluctuations of the water discharge $(Q)$, optical turbidity (optical turbidity), electrical conductivity (Cond), water temperature (temperature), $\delta^{18} \mathrm{O}(\mathrm{dO} 18)$, and $\delta \mathrm{D}(\mathrm{dD})$ in the Djankuat River during the ablation season accompanied by mean daily air temperature (Air temperature), precipitation amount (Prec) and $\delta^{18} \mathrm{O}$ in liquid precipitation (dO18_prec). Drawn using the observational data for June-September 2017.

\subsubsection{Water turbidity}

The regularity of turbidity measurement was defined for each month according to the shape of a diurnal hydrograph of the Djankuat River. The measurement was performed manually using a portable turbidity meter. During heavy rainfall events, the measurements were performed every $15 \mathrm{~min}$ and averaged in the database at an hourly time step. Additionally, water samples were taken manually under various turbidity conditions (in total 19 samples within a range from 66.7 to $36400 \mathrm{NTU}$ ) and filtered using $0.45 \mu \mathrm{m}$ Millipore membrane filters to compute initial suspended sediment concentration (SSC; $\mathrm{mg} \mathrm{L}^{-1}$ ). The values of turbidity were converted to SSC using the abovementioned lab samples using quadratic regression (Belozerova and Chalov, 2013). The total number of 1991 measurements are included in the database (Table 3). The Djankuat River turbidity has an extremely uneven distribution (Fig. 4b): staying less than 400-500 NTU (250$350 \mathrm{~g} \mathrm{~m}^{-3}$ ) most of the time, water turbidity abruptly rose to 1000-5000 NTU (750-4000 $\left.\mathrm{g} \mathrm{m}^{-3}\right)$ and 30000-40 $000 \mathrm{NTU}$ (25000-33000 $\mathrm{g} \mathrm{m}^{-3}$ ) within short periods of heavy rain- fall (more than $20 \mathrm{~mm} \mathrm{~d}^{-1}$ ) (Fig. 6). Observed values close a gap in alpine sediment transport observations and correspond to extreme sedimentation processes registered in the world (e.g., Cohen et al., 2014) and are similar to conditions observed in the most unstable volcanic environments (Chalov et al., 2017b). The maximum value of turbidity (45060 NTU or $37200 \mathrm{~g} \mathrm{~m}^{-3}$ ) was measured on 1 September 2017 after $87 \mathrm{~mm}$ of rain with an average intensity of $30 \mathrm{~mm} \mathrm{~h}^{-1}$. The same rain event triggered an outburst of Bashkara lake in the upstream in a neighboring valley that gave rise to a catastrophic mudflow (Chernomorets et al., 2018).

\subsubsection{Stable isotopes}

Sampling of stable isotope content in the Djankuat River was carried out during 2013-2017 (Table 2a). The concentrations of ${ }^{18} \mathrm{O}$ and $\mathrm{D}$ expressed in the values of $\delta: \delta \mathrm{D}$ $=\left[\left({ }^{2} \mathrm{H} /{ }^{1} \mathrm{H}_{\text {sample }}-{ }^{2} \mathrm{H} /{ }^{1} \mathrm{H}_{\text {standard }}\right) /{ }^{2} \mathrm{H} /{ }^{1} \mathrm{H}_{\text {standard }}\right] \times 1000 \%$; $\delta^{18} \mathrm{O}=\left[\left({ }^{18} \mathrm{O} /{ }^{16} \mathrm{O}_{\text {sample }}-{ }^{18} \mathrm{O} /{ }^{16} \mathrm{O}_{\text {standard }}\right) /{ }^{18} \mathrm{O} /{ }^{16} \mathrm{O}_{\text {standard }}\right]$ $\times 1000 \%$ o. In 2013 all samples were processed in the Stable Isotope Laboratory of the Geography Department of Lomonosov Moscow State University on a Finnigan Delta-V mass spectrometer. For the measurement, international standards were used: V-SMOW (Vienna Standard Mean Ocean Water) with $\delta^{18} \mathrm{O}=0 \%$ and $\delta \mathrm{D}=0 \%$, GISP (Greenland Ice Sheet precipitation) with $\delta^{18} \mathrm{O}=-24.76 \%$ and $\delta \mathrm{D}=$ $-189.5 \%$ o, SLAP (Standard Light Antarctic Precipitation) with $\delta^{18} \mathrm{O}=-55.5 \%$, and our own laboratory standard MSU (Moscow State University) of snow from the Garabashi glacier with $\delta^{18} \mathrm{O}=-15.60 \%$ and $\delta \mathrm{D}=-110.0 \%$. The measurement precision for $\delta^{18} \mathrm{O}$ was $\pm 0.1 \%$.

From 2014 to 2016, all samples were processed in Saint Petersburg State University Resource Center for GeoEnvironmental Research and Modeling (GEOMODEL) on Picarro L-2120i. In 201450 control samples were processed independently by two laboratories: (a) Saint Petersburg State University Resource center for Geo-Environmental Research and Modeling (GEOMODEL) on Picarro L-2120i and (b) the Stable Isotope Laboratory of the Geography Department of Lomonosov Moscow State University on a Finnigan Delta$\mathrm{V}$ mass spectrometer. The difference in the definition for the same sample did not exceed $0.2 \%$.

In 2017, the samples were measured at the Climate and Environmental Research Laboratory (CERL) of the Arctic and Antarctic Research Institute on a laser analyzer Picarro L2140-i that uses the cavity ring-down spectroscopy (CRDS) technique to define the $\delta \mathrm{D}$ and $\delta^{18} \mathrm{O}$ ratios in water samples. After every five samples, we measured our work standard SPB (distilled Saint Petersburg tap water) calibrated against the IAEA standards, in order to obtain accurate values of the samples' isotopic composition. A total of $23 \%$ of randomly chosen samples were re-measured in order to estimate the reproducibility of the results, accordingly $0.06 \%$ for $\delta^{18} \mathrm{O}$ and $0.4 \%$ of $\delta \mathrm{D}$, which is 2 orders of magnitude less than the 
natural variability of the isotopic composition of the studied samples.

In the course of the first test sampling in 2013, a required regularity of sampling to obtain a representative mean daily value of $\delta^{18} \mathrm{O}$ and $\delta \mathrm{D}$ was defined. As the daily variation in $\delta^{18} \mathrm{O}$ and $\delta \mathrm{D}$ turned out to be low compared to other hydrological parameters of the Djankuat River, sampling was performed twice a day in 2014-2017: at the maximum and minimum of water level.

The values of $\delta^{18} \mathrm{O}$ and $\delta \mathrm{D}$ in the Djankuat River waters have a relatively even duration curve (Fig. $4 \mathrm{e}, \mathrm{f}$ ). The value of $\delta^{18} \mathrm{O}$ stayed in the range of $-13.5 \ldots-11.5 \%$ most of the time, and $\delta \mathrm{D}$ stayed in $-95 \ldots-80 \%$. The mean values of $\delta^{18} \mathrm{O}$ and $\delta \mathrm{D}$ are $-12.5 \%$ and $-86.2 \%$ correspondingly. The concentration of ${ }^{18} \mathrm{O}$ and $\mathrm{D}$ decreases with an increase in the share of ice and firn melt in total river flow as shown at the beginning of June and July-August 2017 in Fig. 6. Pronounced rises in $\delta^{18} \mathrm{O}$ and $\delta \mathrm{D}$ were driven by precipitation events (Fig. 6). The maximum value of $\delta^{18} \mathrm{O}$ amounting to $-6.7 \%$ (the content of $\delta \mathrm{D}$ in this sample was $-72 \%$ ) was observed on 16 June 2016 at 22:00 at the beginning of an ablation season after a heavy rain that doubled the mean daily runoff of the Djankuat River. The minimum values of $\delta^{18} \mathrm{O}$ $(-14.7 \%$ o were observed on 17 July 2016 at 21:00 in the course of an intensive snow and ice melting period.

A clear-cut difference in the isotopic composition of ice and snow meltwater and liquid precipitation (see Sect. 3.1.6 of the paper) makes it possible to estimate the ratio of these components to the total river flow. A series of articles were published from the beginning of the 1970s, describing runoff hydrograph separation by nourishment sources with the use of ${ }^{18} \mathrm{O}$ and D (see for example Dincer et al., 1970; Martinec et al., 1974; Fritz et al., 1976; Hermann et al., 1978; Cable, 2011).

A mixing model approach was tested for the Djankuat River to conduct river hydrograph separation in the study (Rets et al., 2017). Two equation systems were drawn: (1) in terms of water routing with salinity as an indicator and (2) in terms of runoff genesis with $\delta^{18} \mathrm{O}$ as a tracer. In terms of water routing the Djankuat River hydrograph was separated into surface-routed and subsurface-routed waters. In terms of runoff genesis the Djankuat River hydrograph was separated into liquid precipitation and meltwater. Some $70 \%$ of the Djankuat River runoff in August-September 2014 was formed by ice and firn meltwater. Rainwater is mostly subsurfacely routed; surface runoff of liquid precipitation is formed only during the most intensive rainfall (more than $20 \mathrm{~mm}$ on average). Ice and firn meltwater partly percolates to the glacier bottom and comes through a subsurface layer. The fast (responsive to weather fluctuations) and regulated components of sub-glacier runoff can be distinguished. Subglacier runoff contributed $20 \%-30 \%$ to the Djankuat River melt runoff in August 2014 and up to $100 \%$ of the Djankuat River melt runoff at the end of September 2014, when ablation stopped (Rets et al., 2017).

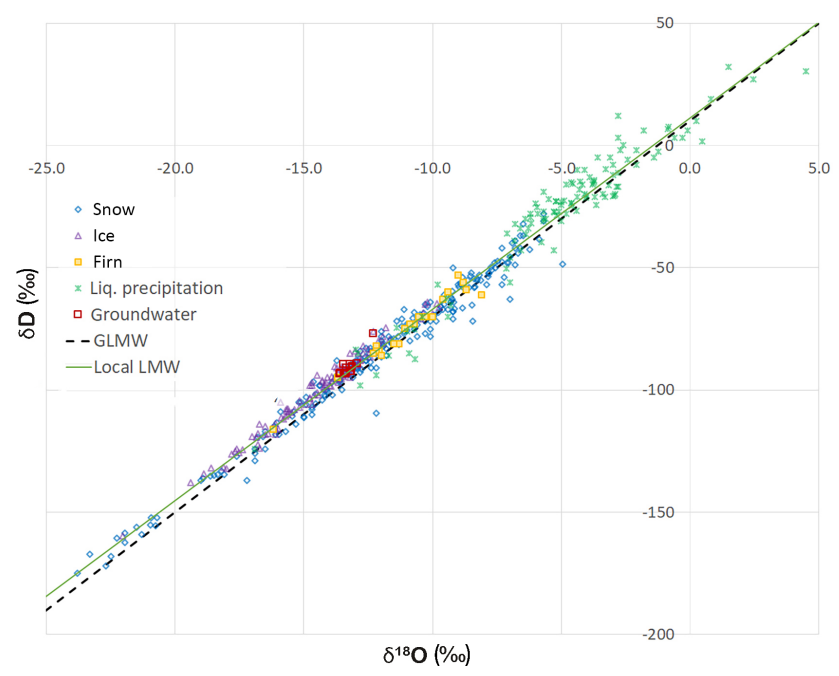

Figure 7. $\delta^{18} \mathrm{O}$ vs. $\delta \mathrm{D}$ graph for the samples of snow, ice, firn, liquid precipitation, and groundwater collected in the Djankuat River basin in 2013-2017, plotted with a global and local meteoric water line.

\subsubsection{Sampling snow, ice, firn, liquid precipitation, and groundwater in the basin for stable isotopes and electrical conductivity}

Regular sampling of snow, ice, firn, liquid precipitation, and groundwater in the Djankuat basin was carried out during 2014-2017. The first test sampling was performed in 2013. Liquid precipitation was sampled on every significant occasion of rainfall (more than $1 \mathrm{~mm}$ ) and amounts to 25-30 samples a year at the base camp weather station (Table 1). The snow, ice, and firn sampling points were evenly distributed in the Djankuat basin area; the coordinates are given in the database. Samples were taken regularly during the ablation season, 40-150 samples a year. Snow samples were taken from the surfaces at different depths of the snowpack. The groundwater was sampled (1) after the end of ablation season in the Djankuat River stream when the total runoff is assumed to be provided by groundwater in 2014, (2) out of a subglacial water spring in 2015, and (3) out of a groundwater-fed spring on the slope of the Djankuat River basin in 2017.

In total 113 samples of liquid precipitation, 218 samples of snow, 116 samples of ice, 22 samples of firn, and 16 samples of groundwater were taken. The values of $\delta^{18} \mathrm{O}$ and $\delta \mathrm{D}$ were measured in each sample. The proceeding of the samples was the same for the stable isotope samples taken at the gauging station (see Sect. 3.1.5). Relative concentrations of ${ }^{18} \mathrm{O}$ and $\mathrm{D}$ in precipitation are strongly correlated and defined to a great extent by air temperature, which is called the "seasonal isotope phenomenon" (Dansgaard, 1964). The highest concentrations of ${ }^{18} \mathrm{O}$ and $\mathrm{D}$ among the samples collected in the Djankuat River basin are characteristic of liquid precipitation (Table 3, Fig. 7). The $\delta^{18} \mathrm{O}$ value lies mostly between $-0.5 \%$ and $-7 \%$ with a mean value of $-4.9 \%$. The 


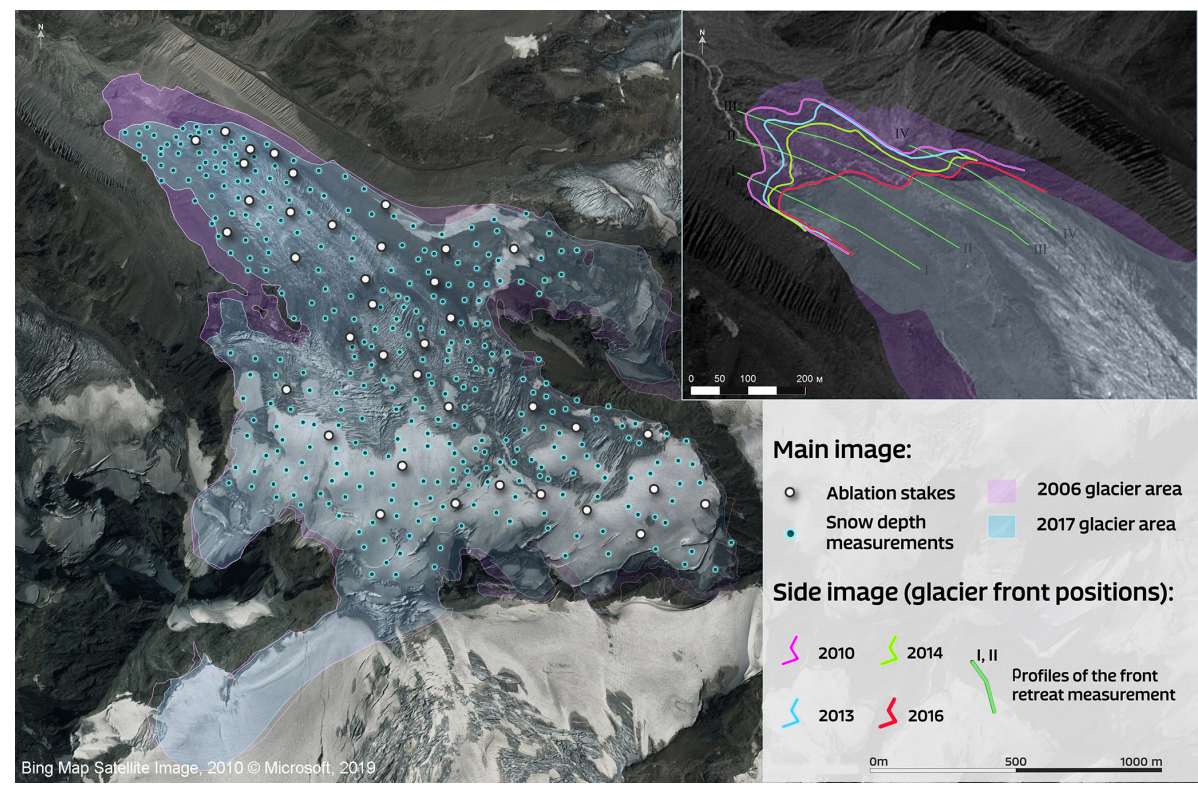

Figure 8. The spatial distribution of ablation stakes and snow thickness measurement points, change in the Djankuat glacier area during the study period, and glacier front position.

$\delta \mathrm{D}$ value lies between $0 \%$ and $-40 \%$, and the mean value is $-26 \%$ o. The lowest concentrations of ${ }^{18} \mathrm{O}$ and $\mathrm{D}$ were registered in winter snow $\left(\delta^{18} \mathrm{O}=-28.3 \%\right.$ o, $\delta \mathrm{D}=-216 \%$ ) and Djankuat glacier ice $\left(\delta^{18} \mathrm{O}=-22.0 \%, \delta \mathrm{D}=-159 \%\right.$ o $)$. The number of stable isotopes in snow cover formed during spring snowfalls is higher and closer to the corresponding values in liquid precipitation $\left(\delta^{18} \mathrm{O}=-9 \ldots-5 \%\right.$, $\delta \mathrm{D}=-28 \ldots-70 \%$ ). The mean concentration of ${ }^{18} \mathrm{O}$ and $\mathrm{D}$ is quite similar: the mean $\delta^{18} \mathrm{O}$ is $-12.2 \%$ in snow samples, $-14.3 \%$ in ice samples, and $-11.3 \%$ in firn; mean $\delta \mathrm{D}$ is $-85.5 \%$ o, $-99.3 \%$, and $-77.1 \%$ o, respectively. The surface of a glacier ablation area is always formed by ice layers of different ages, while firn samples represent the climatic conditions of the last 5-10 years. A generally warmer mean isotopic composition of firn and snow compared to ice can indicate total warming of the climate. Groundwater is a mixture of all the above-stated sources. Accordingly, the points of groundwater samples lie in the middle of the $\delta^{18} \mathrm{O}$ vs. $\delta$ D graph (Fig. 7). The mean concentration of ${ }^{18} \mathrm{O}$ and $\mathrm{D}$ in groundwater samples $(-13.3 \%$ and $-91 \%$ correspondingly) indicates a bigger role of meltwater than of summer precipitation in the replenishment of groundwater layers.

The ice, snow, and firn samples are ultra-fresh (Table 3). Groundwater is enriched with dissolved salts up to $105 \mathrm{mg} \mathrm{L}^{-1}\left(114 \mu \mathrm{Sm} \mathrm{cm}^{-1}\right)$.

\subsection{Glaciological measurements}

Glaciological observations were carried out using standard methods during 2007-2017 (Østrem and Brugman, 1991;
Boyarsky, 1978). The data on snow depth, snow density, and ablation are included in the presented database (Table $2 b$ ).

Snow depth on the Djankuat glacier was measured at 250 300 points evenly distributed in all zones of the glacier (Fig. 8). The snow measurement survey usually started in late May-early June and ended at the end of June. The mean value of measured snow thickness was $3.6 \mathrm{~m}$, and the maximum was $11.5 \mathrm{~m}$.

Snow density was measured in two to four snow pits placed in different elevation belts of the Djankuat glacier (Fig. 1). Density was measured in each $40-50 \mathrm{~cm}$ layer of a snowpack (Fig. 3d). The measurements were repeated two to five times during the ablation season. The integral density of snowpack had a low variance; total range of variation was less than $0.2 \mathrm{~g} \mathrm{~cm}^{-3}$. The density in the layers of the snowpack greatly varied - from 0.23 to $0.92 \mathrm{~g} \mathrm{~cm}^{-3}$ according to the 2007-2017 measurements. The overall mean measured value of snow density in the snowpack, $0.57 \mathrm{~g} \mathrm{~cm}^{-3}$, greatly exceeds the density of fresh snow, as the database includes measurements carried out in midsummer and at the end of the ablation period (Table 3 ).

A total of 35-45 ablation stakes were placed on the Djankuat glacier surface every year (Fig. 8). The time step between measurements depended on the accessibility of each stake and ranged from 1-5 to $30 \mathrm{~d}$. The values included in the dataset are counted from the measured depth of melted snow-firn-ice in centimeters and corresponding values of density of melting material.

The Djankuat glacier has experienced a general mass loss since the beginning of observation in 1968 (https://wgms. ch/, last access: 13 September 2019). Until 2005, nega- 


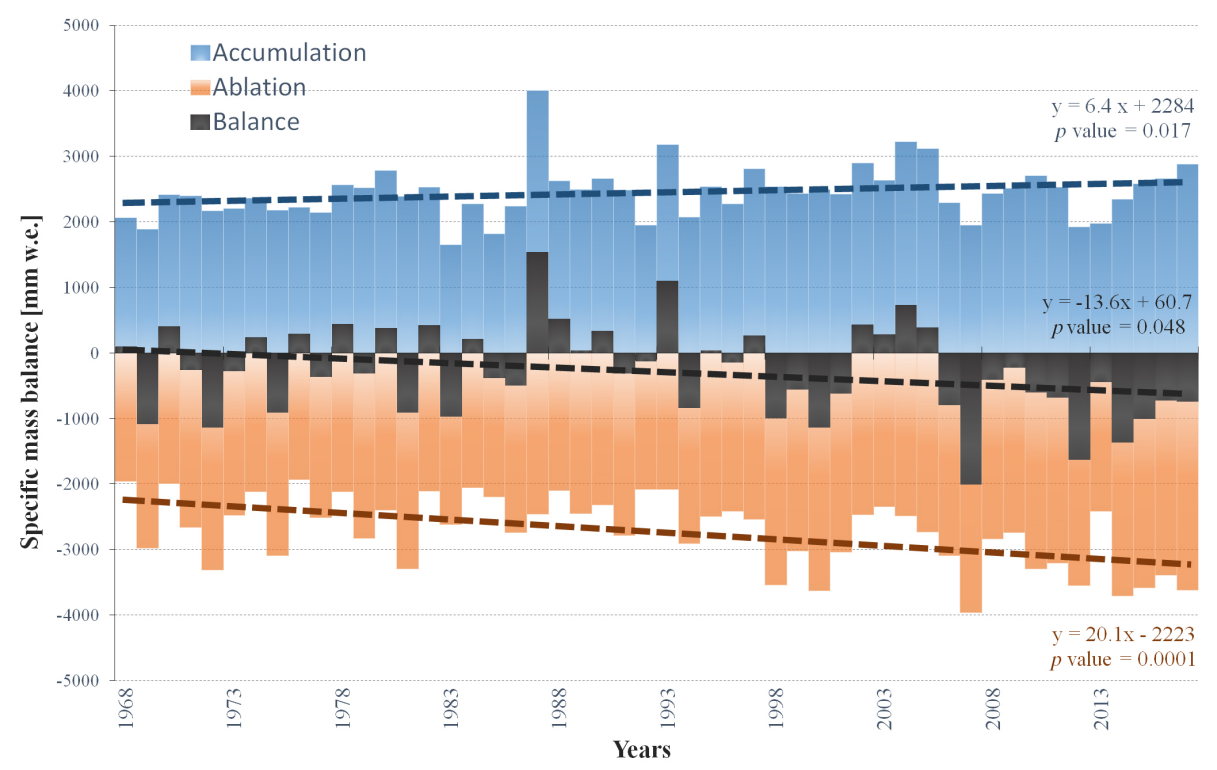

Figure 9. Fluctuations of the Djankuat glacier mass balance components in millimeters of water equivalent (mm w.e.) since the beginning of the observations in 1968 (https://wgms.ch/, last access: 13 September 2019).

tive mass balance years alternated with positive mass balance years (Fig. 9). After 2006 and during all periods that were presented in the dataset, the annual mass balance of Djankuat glacier was negative. The annual mass balance values ranged from $-2010 \mathrm{~mm}$ w.e. in 2007, which was the lowest value since the beginning of the observation in 1968, to $-230 \mathrm{~mm}$ w.e. in 2009 . The mean value of annual mass balance during 2007-2017 was -900 mm w.e. During 20072017 the area of the Djankuat glacier decreased from 2.68 to $2.42 \mathrm{~km}^{2}$, which amounts to almost $10 \%$ of loss (Fig. 8). The front of the glacier retreated by $60-300 \mathrm{~m}$ in different measurement profiles during 2010-2016 (Fig. 8), which amounts to $8.5-42.9 \mathrm{~m} \mathrm{yr}^{-1}$ retreatment rate. The main reason for the Djankuat glacier retreating during the long-term period is an intensive decrease in summer balance, while accumulation shows a slightly positive trend (Fig. 9).

The information on Djankuat glacier mass balance, calculated from the presented dataset, is being published in the "Glacier Mass Balance Bulletin", which is issued by the World Glacier Monitoring Service (WGMS) at 2-year intervals and in "Fluctuations of Glaciers" along with other standardized data on changes in glaciers throughout the world at 5-year intervals (see for example Popovnin, 2012, 2013).

Some of the glacial measurement data presented in this article were used in global studies on evolution of the Earth's cryosphere (Zemp et al., 2009, 2011, 2015). In Popovnin and Pylayeva (2015) snow thickness measurements on Djankuat glacier were used to work out a methodology of estimation of avalanche feeding of a glacier from the total mass of snow accumulation.

\subsection{Meteorological measurements}

The main purpose of meteorological measurements in the Djankuat research basin is to provide the data needed for calculating the components of the heat balance (Toropov et al., 2017, 2018b), which is a necessary input for physically based hydrological models. As an example, the presented meteorological data were successfully used to model the melting regime of the Djankuat glacier in 2007 by the A-Melt model of ice melt and snowmelt in alpine areas (Rets and Kireeva, 2010).

The program of meteorological observations in the Djankuat research basin during 2007-2017 included meteorological and radiation measurements by means of two Campbell AWSs placed above the ice surface (Gjankuat glacier AWS 1 in Tables 1-2, Figs. 1, 3a) and over the debriscovered surface (Gjankuat glacier AWS 2 in Tables 1-2, Fig. 1). In order to measure the ablation rate at both sites by a Sonic Ranger, the sensor was located on a construction drilled into the body of the glacier and measured the distance from the sensor to the ice or snow surface. Standard meteorological characteristics were monitored at the base camp by means of a Davis AWS (base camp AWS in Tables 1-2, Figs. 1, 3c). In 2017 a Davis AWS was also placed in the upper part of the glacier (Djankuat glacier AWS 3 in Tables 1-2, Fig. 1). 


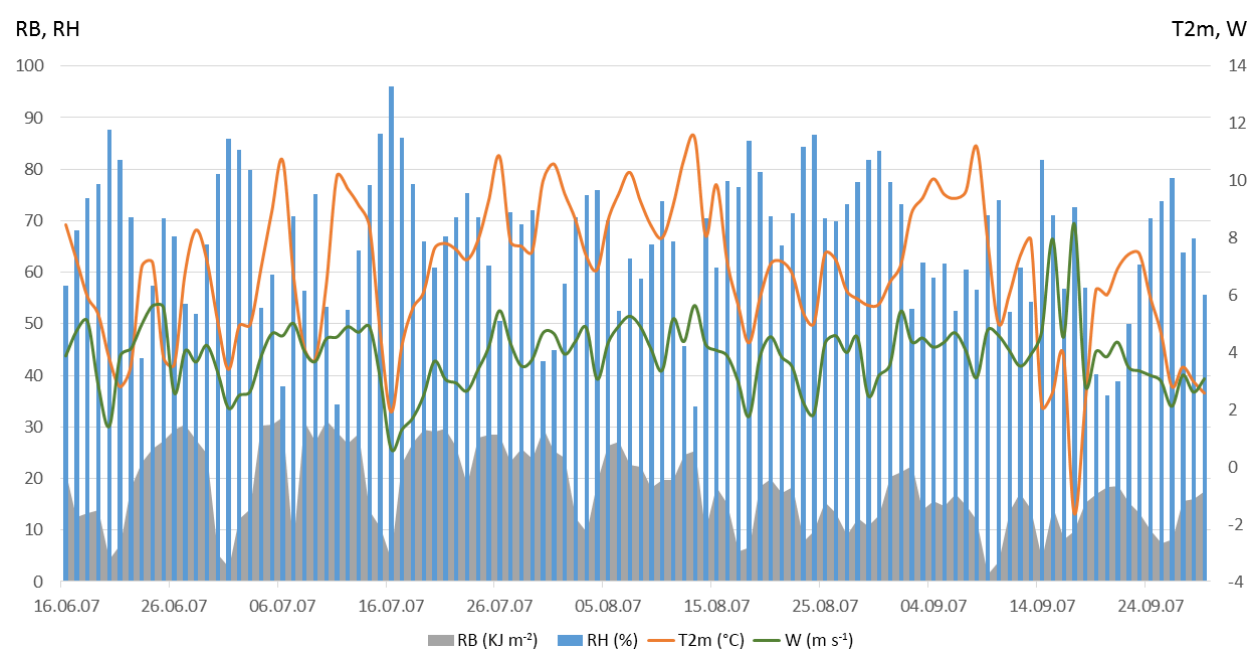

Figure 10. An example of temporal variability of average daily values on the Djankuat glacier (measured at Djankuat glacier AWS 1 location) in 2007: $\mathrm{RB}$ - radiation balance, $\mathrm{KJ} \mathrm{m}^{-2}$; $\mathrm{RH}$ - relative humidity; $\% \mathrm{~T} 2 \mathrm{~m}$ - air temperature, ${ }^{\circ} \mathrm{C} ; W$ - wind speed, $\mathrm{m} \mathrm{s}^{-1}$.

Table 4. July daily averaged meteorological variables on Djankuat glacier from 2007 to 2017 (with rms in brackets).

\begin{tabular}{|c|c|c|c|c|c|c|c|c|c|c|c|c|}
\hline \multirow[t]{2}{*}{ Year } & \multicolumn{3}{|c|}{ Air temperature, ${ }^{\circ} \mathrm{C}$} & \multicolumn{2}{|c|}{ Relative humidity, \% } & \multicolumn{2}{|c|}{ Wind speed, $\mathrm{m} \mathrm{s}^{-1}$} & \multicolumn{5}{|c|}{ Components of radiation balance, $\mathrm{W} \mathrm{m}^{-2}$ Albedo $A, \%$} \\
\hline & Mean & Min & Max & Mean & Min & Mean & Max & SW+ & SW- & $\mathrm{LW}+$ & LW- & $A, \%$ \\
\hline 2007 & $8.0( \pm 2.6)$ & 0.4 & 13.5 & $66( \pm 19)$ & 13 & $3.8( \pm 1.7)$ & 8.4 & $247( \pm 99)$ & $68( \pm 39)$ & $280( \pm 27)$ & $314( \pm 3)$ & 19 \\
\hline 2008 & $8.1( \pm 2.1)$ & 2.3 & 13.9 & $72( \pm 15)$ & 24 & $4.2( \pm 1.8)$ & 9.3 & $237( \pm 105)$ & $88( \pm 58)$ & $291( \pm 26)$ & $315( \pm 4)$ & 32 \\
\hline 2009 & $6.0( \pm 2.5)$ & -0.5 & 14.2 & $76( \pm 13)$ & 36 & $3.8( \pm 1.7)$ & 9.0 & $225( \pm 88)$ & $71( \pm 48)$ & $286( \pm 29)$ & $313( \pm 8)$ & 23 \\
\hline 2010 & $8.3( \pm 2.2)$ & 2.9 & 15.2 & $68( \pm 14)$ & 31 & $4.2( \pm 1.3)$ & 8.5 & $265( \pm 84)$ & $43( \pm 15)$ & $293( \pm 21)$ & $317( \pm 5)$ & 18 \\
\hline 2012 & $7.7( \pm 2.0)$ & 1.7 & 15.2 & $71( \pm 15)$ & 31 & $3.9( \pm 1.6)$ & 7.9 & $267( \pm 104)$ & $57( \pm 25)$ & $290( \pm 19)$ & $323( \pm 3)$ & 21 \\
\hline 2013 & $5.0( \pm 2.2)$ & -0.7 & 10.7 & $77( \pm 12)$ & 40 & $3.5( \pm 2.0)$ & 10.5 & $225( \pm 98)$ & $53( \pm 30)$ & $300( \pm 22)$ & $325( \pm 4)$ & 24 \\
\hline 2014 & $7.6( \pm 2.1)$ & 2.4 & 14.7 & $67( \pm 16)$ & 18 & $3.6( \pm 1.6)$ & 8.3 & $274( \pm 111)$ & $47( \pm 18)$ & $306( \pm 18)$ & $293( \pm 6)$ & 19 \\
\hline 2015 & $8.8( \pm 2.8)$ & -0.1 & 17.9 & $65( \pm 17)$ & 15 & $4.0( \pm 1.8)$ & 8.9 & $308( \pm 78)$ & $75( \pm 22)$ & $357( \pm 10)$ & $332( \pm 5)$ & 24 \\
\hline 2016 & $7.6( \pm 2.8)$ & 0.2 & 15.1 & $69( \pm 16)$ & 24 & $3.8( \pm 1.9)$ & 9.0 & $235( \pm 92)$ & $65( \pm 23)$ & $305( \pm 15)$ & $315( \pm 6)$ & 23 \\
\hline 2017 & $8.3( \pm 2.3)$ & 0.5 & 17.6 & $63( \pm 15)$ & 20 & $4.2( \pm 2.0)$ & 10.1 & $224( \pm 98)$ & $54( \pm 30)$ & $301( \pm 22)$ & $324( \pm 4)$ & 25 \\
\hline Mean & $7.6( \pm 2.3)$ & 1.1 & 14.5 & $68( \pm 17)$ & 25 & $3.9( \pm 1.7)$ & 8.9 & $231( \pm 94)$ & $63( \pm 25)$ & $300( \pm 10)$ & $317( \pm 5)$ & 24 \\
\hline
\end{tabular}

A number of experimental observations were carried out at the Gjankuat glacier AWS 1: gradient observations to obtain turbulent heat flux estimations with the Monin-Obukhov method in 2015, which included four temperature and humidity sensors and four wind sensors located at 0.25, 0.5, 1, and $2 \mathrm{~m}$ above the glacier surface, and measurements of highfrequency turbulent pulsations of wind and acoustic temperature (Fig. 3c).

Figure 10 shows an example of the course of the average daily values of the basic meteorological variables during the ablation season of 2007 measured by Campbell AWS 1. It is clearly seen that changes in air temperature associated with synoptic events are expressed quite well, their average amplitude being $3{ }^{\circ} \mathrm{C}$. The average temperature during the ablation period was around $8^{\circ} \mathrm{C}$, while the minimum values almost reached $0^{\circ} \mathrm{C}$ annually, and the maximums were 16 to $18^{\circ} \mathrm{C}$. The variability of the radiation balance is determined mainly by cloudiness, which primarily has a pronounced daily variation. The albedo effect was also clearly manifested - espe- cially in June and September when fresh snow frequently fell on the surface of the glacier. The maximum values of incoming shortwave radiation can reach $1100 \mathrm{~W} \mathrm{~m}^{-2}$. The wind regime is stable to a great extent and varies little from year to year. The average wind speed over the tongue of the glacier was fairly stable at $4-6 \mathrm{~m} \mathrm{~s}^{-1}$. Above the glacier, stable katabatic winds blow, which is characterized by a pronounced diurnal course. The most probable value of the maximum gusts was $10-12 \mathrm{~m} \mathrm{~s}^{-1}$. The absolute maximum for the period under review was $16.6 \mathrm{~m} \mathrm{~s}^{-1}$ above the surface of the glacier and $21 \mathrm{~m} \mathrm{~s}^{-1}$ above the moraine ridge. The strongest glacial wind was observed in the lower part of the glacier, in the area of the weather station AWS 1, where the maximum slope angle and density contrast between glacial and valley air were combined. The wind decreases down the valley, and in the area of the base camp its gusts rarely exceeded $10 \mathrm{~m} \mathrm{~s}^{-1}$. The maximum speed values were associated with foehn winds, observed three to four times per season. Table 4 shows the 


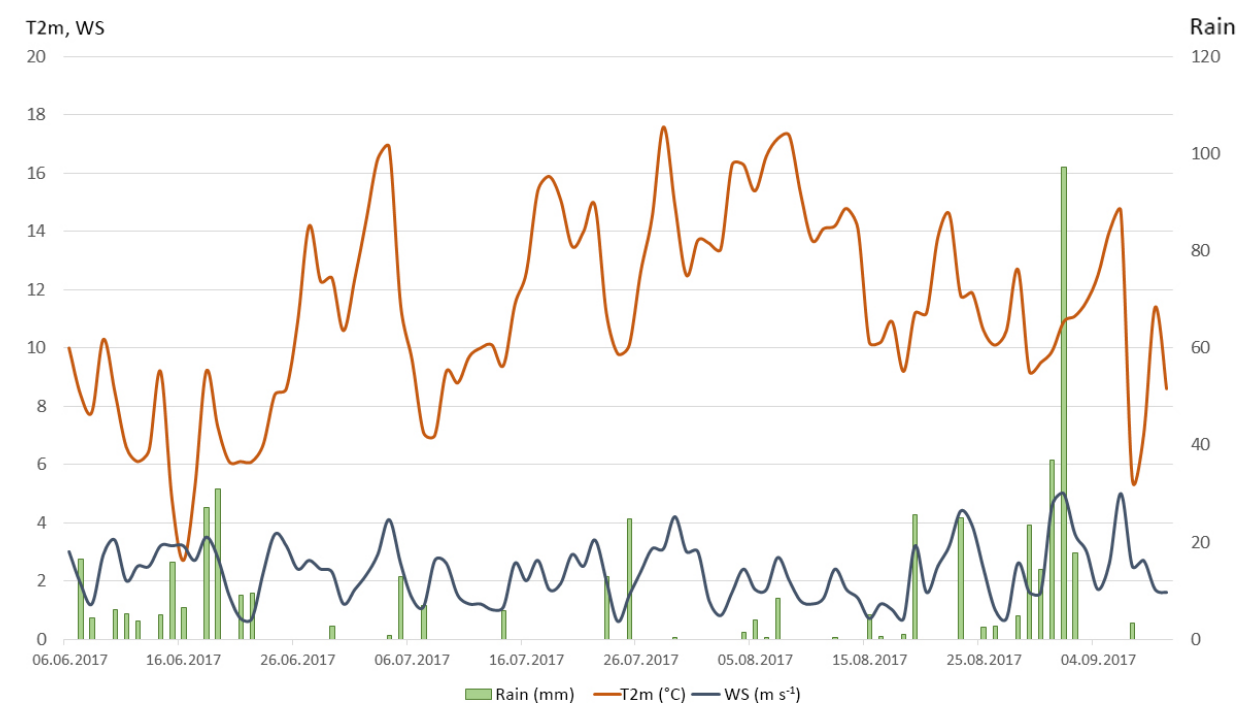

Figure 11. An example of temporal variability of average daily temperature, wind speed, and precipitation sum at the base camp (point AWS - "base camp"): Rain - precipitation, $\mathrm{mm} \mathrm{d}^{-1}$; $\mathrm{T} 2 \mathrm{~m}$ - air temperature, ${ }^{\circ} \mathrm{C}$; WS - wind speed, $\mathrm{m} \mathrm{s}^{-1}$.

average July values of the main meteorological characteristics.

Figure 11 gives an example of the variability of the main meteorological characteristics in the Djankuat River valley, in the area of the base camp at an altitude of $2640 \mathrm{~m}$ above sea level (Table 1, Fig. 1). The average daily temperature is about $12^{\circ} \mathrm{C}$, and the average wind speed is about $2 \mathrm{~m} \mathrm{~s}^{-1}$, even though the maximum gusts were stronger over the glacier and reach $18 \mathrm{~m} \mathrm{~s}^{-1}$. This is due to the density difference between the cold air flowing from the glacier and the local air mass forming over the heated alpine meadows and rocks. Heavy rains can be observed in the study area. For example, in 2017, the daily precipitation of about $20 \mathrm{~mm}$ was observed six times. From 31 August to 1 September, about $48 \mathrm{~mm}$ of precipitation fell within $48 \mathrm{~h}$, which is a very high amount. This rainfall caused the abovementioned outburst of the Bashkara glacial lake in the neighboring valley and the formation of a catastrophic mudflow (Chernomorets et al., 2018).

\section{Data availability}

The presented datasets are available open access via the PANGAEA repository (https://doi.org/10.1594/PANGAEA.894807) (Rets et al., 2018a).

\section{Conclusion}

With the detailed measurement program described here, the Djankuat basin is expected to serve as a highly valuable benchmark for evaluating, calibrating, and further developing glaciological and hydro-sedimentological catchment models of the alpine catchments of the North Caucasus and Russian Federation as a whole (Konovalov et al., 2018; Stokes et al., 2006; Shahgedanova et al., 2005; Hagg et al., 2010). The aim of the multidisciplinary monitoring in the Djankuat basin is not only to fill a blind spot in extremely underreported North Caucasus alpine territories but to provide data for detailed studies of hydrometeorological processes in mountain areas (Rets et al., 2017; Toropov et al., 2017).

The dataset presented here covers the period of 2007-2017 and can be useful to researchers developing and verifying hydrological, glaciological, and meteorological models for mountainous territories, studying the recent climate and its impact on the cryosphere and hydrology, and using isotopic and hydrochemical approaches to study the source areas of runoff.

Author contributions. EPR led the hydrological data collection and proceeding, created the database, prepared the paper. VVP organized the Djankuat station operation and led glaciological data collection. PAT led meteorological data collection and preparation and contributed to paper sections. AMS prepared glaciological data and contributed to preparation of graphical materials. IVT, JNC, $\mathrm{NAB}, \mathrm{AAE}, \mathrm{ANV}$, and $\mathrm{YKV}$ performed the staple isotope analyses. MBK and NLF contributed to organization of hydrological monitoring and contributed to hydrological data collection. AST contributed to the design and organization of water turbidity monitoring and preparation of water turbidity data. SRC provided guidance on and contributed to organization and water turbidity measurements. AAP and MAA contributed to collection and preparation of meteorological data. EDK contributed to collection and preparation of hydrological data. AAA provided information on the Djankuat glacier front movement. 
Competing interests. The authors declare that they have no conflict of interest.

Disclaimer. The data are provided with no warranty.

Special issue statement. This article is part of the special issue "Hydrometeorological data from mountain and alpine research catchments". It is not associated with conference.

Acknowledgements. This work was supported by the Russian Foundation for Basic Research (project no. 16-35-60042 - part of hydrological observations, project no. 18-05-00420 - part of glaciological observations, 17-05-00771 - part of meteorological observations, project no. 18-05-60272 - part of isotope analysis) and the grant of the president of the Russian Federation for young scientists MK-2936.2019.5 - part of the Djankuat glacier mass balance dynamics analysis.

We want to give our gratitude to all members of the glacial party of Moscow State University, who were working at the Djankuat glacier station during 2007-2017, amounting to more than 100 people in total. It would not have been possible to collect the data presented in this paper if it were not for them. In particular we would like to thank Fedor Andryushchenko, Elena Astafyeva, Anna Avilova, Varvara Bazilova, Egor Belozerov, Victorya Bychkova, Vasily Efimov, Natalia Ezerova, Maria Gaydamukha, Aphanasy Gubanov, Valera Ivanov, Maria Kaminskaya, Nickolay Kovalenko, Ekaterina Kuzmina, Nadezhda Loshakova, Pavel Lysenok, Ilya Marchuk, Tatiana Matveeva, Polina Morozova, Evgenia Panchenko, Valentin Pastukhov, Alexey Rezepkin, Alexander Rostiashvili, Yana Sergievskaya, Anna Shestakova, Maria Tereshina, Denis Zakharhenko, and Irina Zheleznova.

Financial support. This research has been supported by the Russian Foundation for Basic Research (grant nos. 16-35-60042, 1805-00420, and 17-05-00771) and the grant of the president of the Russian Federation for young scientists MK-2936.2019.5.

Review statement. This paper was edited by John Pomeroy and reviewed by Ludwig Braun and one anonymous referee.

\section{References}

Alekseev, G. V., Ananicheva, M. D., Anisimov, O. A., Ashik, I. M., Bardin, M. Y., and Bogdanova, E. G.: The second assessment report of Roshydromet on climate change and its consequences on the territory of the Russian Federation, State Scientific Center of the Russian Federation "Arctic and Antarctic Research Institute" Federal Service of Russia for Hydrometeorology and Environmental Monitoring, Moscow, Russia, 2014

Auer, I., Böhm, R., Jurkovic, A., Lipa, W., Orlik, A., Potzmann, R., Schöner, W., Ungersböck, M., Matulla, C., Briffa, K., Jones, P., Efthymiadis, D., Brunetti, M., Nanni, T., Maugeri,
M., Mercalli, L., Mestre, O., Moisselin, J., Begert, M., MüllerWestermeier, G., Kveton, V., Bochnicek, O., Stastny, P., Lapin, M., Szalai, S., Szentimrey, T., Cegnar, T., Dolinar, M., GajicCapka, M., Zaninovic, K., Majstorovic, Z., and Nieplova, E.: HISTALP - historical instrumental climatological surface time series of the Greater Alpine Region, Int. J. Climatol., 27, 17-46, https://doi.org/10.1002/joc.1377, 2007.

Bales, R. C., Molotch, N. P., Painter, T. H., Dettinger, M. D., Rice, R., and Dozier, J.: Mountain hydrology of the western United States, Water Resour. Res., 42, W08432, https://doi.org/10.1029/2005WR004387, 2006.

Barry, R. G.: Mountain Climatology and Past and Potential Future Climatic Changes in Mountain Regions: A Review, Mt. Res. Dev., 12, 71-86, https://doi.org/10.2307/3673749, 1992.

Belozerova, E. V. and Chalov, S. R. Assessment of river water turbidity using the optic methods, Vestnik Moskovskogo Universiteta, Seriya 5, Geografiya, 6, 39-45, 2013.

Bobrovitskaya, N. N. and Kokorev, A. V.: Current problems of hydrological networks design and optimization, Background material for the fourteenth session of the Commission for Hydrology (CHy-14), Geneva, Switzerland, 2014.

Boyarsky, I. Y. (Ed.): Lednik Djankuat (Djankuat Glacier), Gidrometeoizdat, Saint Petersburg, Russia, 1978.

Cable, J., Ogle, K., and Williams, D.: Contribution of glacier meltwater to streamflow in the Wind River Range, Wyoming, inferred via a Bayesian mixing model applied to isotopic measurements, Hydrol. Process., 25, 2228-2236, https://doi.org/10.1002/hyp.7982, 2011.

Chalov, S., Golosov, V., Tsyplenkov, A., Theuring, P., Zakerinejad, R., Märker, M., and Samokhin, M.: A toolbox for sediment budget research in small catchments, Geography, Environment, Sustainability, 10, 43-68, https://doi.org/10.24057/2071-9388-201710-4-43-68, 2017a.

Chalov, S. R., Tsyplenkov, A. S., Pietron, J., Chalova, A. S., Shkolny, D. I., Jahsjo, J., and Maerker, M.: Sediment transport in headwaters of a volcanic catchment - Kamchatka Peninsula case study, Front. Earth Sci. Frontiers of Earth Science 11, 565578, https://doi.org/10.1007/s11707-016-0632-x, 2017b.

Chernomorets, S. S., Petrakov, D. A., Aleynikov, A. A., Bekkiev, M. Y., Viskhadzhieva, K. S., Dokukin, M. D., Kalov, R. K., Kidyaeva, V. M., Krylenko, V. V., Krylenko, I. V., Krylenko, I. N., Rets, E. P., Savernyuk, E. A., and Smirnov, A. M.: The outburst of Bashkara glacier lake (central Caucasus, Russia) on september 1, 2017, Earth's Cryosphere, 22, 70-80, https://doi.org/10.21782/kz1560-7496-2018-2(70-80), 2018.

Cohen, S., Kettner, A. J., and Syvitski, J. P. M.: Global suspended sediment and water discharge dynamics between 1960 and 2010: Continental trends and intra-basin sensitivity, Global Planet. Change, 115, 44-58, 2014.

Dansgaard, W.: Stable isotopes in precipitation, Tellus, 16, 436468, 1964.

Dincer, T., Payne, B. R., Flowkowski, T., Martinec, J., and Tongiorgi, E.: Snowmelt runoff from measurements of tritium and oxygen-18, Water Resour. Res., 6, 110-124, https://doi.org/10.1029/WR006i001p00110, 1970.

Dobriyal, P., Badola, R., Tuboi, C., and Hussain, S. A.: A review of methods for monitoring streamflow for sustainable water resource management, Appl. Water Sci., 7, 2617-2628, https://doi.org/10.1007/s13201-016-0488-y, 2017. 
Dyurgerov, M.: Mountain and subpolar glaciers show an increase in sensitivity to climate warming and intensification of the water cycle, J. Hydrol., 282, 164-176, https://doi.org/10.1016/S00221694(03)00254-3, 2003.

Fritz, P., Cherry, J., Weyer, K., and Sklash, M.: Storm runoff analyses using environmental isotopes and major ions, in: Interpretation of Environmental Isotope and Hydrochemical Data in Groundwater, Panel Proc. Ser. - Int. Atomic Energy Agency, Vienna, Int. Atomic Energy Agency, 111-130, 1976.

Gietl, G.: Collection and processing of hydrometeorological and hydrological data in mountainous areas, Hydrology of Mountainous areas, Proceedings of the atrbske Pleso Workshop, Czechoslovakia, June 1988, IAHS P., 190, 3-12, 1990.

Hagg, W., Shahgedanova, M., Mayer, C., Lambrecht, A., and Popovnin, V.: A sensitivity study for water availability in the Northern Caucasus based on climate projections, Global Planet. Change, 73, 161-171, https://doi.org/10.1016/j.gloplacha.2010.05.005, 2010.

Hermann, A., Martinec, J., and Stichler, W.: Study of snowmeltrunoff components using isotope measurements, in: Proceedings of Modeling of Snow Cover Runoff, edited by: Colbeck, S. C. and Ray, M., U.S. Army CRREL Special Report, 79-36, 288296, 1978.

Immerzeel, W. W., van Beek, L. P. H., Konz, M., Shrestha, A. B., and Bierkens M. F. P.: Hydrological response to climate change in a glacierized catchment in the Himalayas, Climatic Change, 110, 721-736, https://doi.org/10.1007/s10584011-0143-4, 2012.

Konovalov, V. G., Rets, E. P., and Pimankina, N. A.: Hydrological essense of glacier's summer mass balance in mountain river basins, Collective monograph on materials of the annual International scientific and practical conference LXXI Gertsenovsky readings, to the 155 anniversary since the birth of Vladimir Ivanovich Vernadsky, Geography: development of science and education, 1, St. Petersburg, RSPU of A.I. Herzen, 1821 April 2018, edited by: Solomin, V. P., Rumyantsev, V. A., Subetto, D. A., and Lovelius, N. V., St. Petersburg, RSPU of name A. I. Herzen pubishing house, Russia, 2018.

Lambrecht, A., Mayer, C., Hagg, W., Popovnin, V., Rezepkin, A., Lomidze, N., and Svanadze, D.: A comparison of glacier melt on debris-covered glaciers in the northern and southern Caucasus, The Cryosphere, 5, 525-538, https://doi.org/10.5194/tc-5525-2011, 2011.

Lavrentiev, I. I., Kutuzov, S. S., Petrakov, D. A., Popov, G. A., and Popovnin, V. V.: Ice thickness, volume and subglacial relief of Djankuat Glacier (Central Caucasus), Ice and Snow, 54, 7-19, https://doi.org/10.15356/2076-6734-2014-4-7-19, 2014.

Martinec, J., Siegenthaler, U., Oeschger, H., and Tongiorgi, E.: New insights into the run-off mechanism by environmental isotopes, Proc. Sympos. Isotope Tech. in Groundwater Hydrol., Vienna, Int. Atomic Energy Agency, 4, 129-143, RN:38048670, 1974.

Meier, M. F., Dyurgerov, M. B., and McCabe, G. J.: The Health of Glaciers: Recent Changes in Glacier Regime, Climatic Change, 59, 123-135, https://doi.org/10.1023/A:1024410528427, 2003.

Østrem, G. and Brugman, M.: Glacier Mass-Balance Measurements, A manual for field and office work, NHRI Science Report No. 4, 1991.

Pachauri, R. K., Allen, M. R., Barros, V. R., Broome, J., Cramer, W., Christ, R., Church, J. A., Clarke, L., Dahe, Q., Dasgupta, P.,
Dubash, N. K., Edenhofer, O., Elgizouli, I., Field, C. B., Forster, P., Friedlingstein, P., Fuglestvedt, J., Gomez-Echeverri, L., Hallegatte, S., Hegerl, G., Howden, M., Jiang, K., Jimenez Cisneroz, B., Kattsov, V., Lee, H., Mach, K. J., Marotzke, J., Mastrandrea, M. D., Meyer, L., Minx, J., Mulugetta, Y., O’Brien, K., Oppenheimer, M., Pereira, J. J., Pichs-Madruga, R., Plattner, G. K., Pörtner, H. O., Power, S. B., Preston, B., Ravindranath, N. H., Reisinger, A., Riahi, K., Rusticucci, M., Scholes, R., Seyboth, K., Sokona, Y., Stavins, R., Stocker, T. F., Tschakert, P., van Vuuren, D., and van Ypserle, J. P.: Climate Change 2014: Synthesis Report. Contribution of Working Groups I, II and III to the Fifth Assessment Report of the Intergovernmental Panel on Climate Change, edited by: Pachauri, R. and Meyer, L., Geneva, Switzerland, IPCC, 151, ISBN 978-92-9169-143-2, 2014.

Popovnin, V. V.: Djankuat, Fluctuations of Glaciers, 10, 196-197, 2012.

Popovnin, V. V.: Djankuat, Glacier Mass Balance Bulletin, 12, 8989, 2013.

Popovnin, V. V. and Pylayeva, T. V.: Avalanche feeding of the Djankuat Glacier, Ice and Snow, 55, 21-32, https://doi.org/10.15356/2076-6734-2015-2-21-32, 2015.

Rets, E. and Kireeva, M.: Hazardous hydrological processes in mountainous areas under the impact of recent climate change: case study of Terek River basin, Global Change: Facing Risks and Threats to Water Resources: proc. of the Sixth World FRIEND Conference, IAHS P., 340, 126-134, 2010.

Rets, E., Chizhova, J. N., Loshakova, N., Tokarev, I., Kireeva, M. B., Budantseva, N. A., Vasil'chuk, Y. K., Frolova, N., Popovnin, V., Toropov, P., Terskaya, E., Smirnov, A. M., Belozerov, E., and Karashova, M.: Using isotope methods to study alpine headwater regions in the northern Caucasus and Tien Shan, Front. Earth Sci., 11, 531-543, https://doi.org/10.1007/s11707-017-0668-6, 2017.

Rets, E. P., V., Toropov, P., Smirnov, A., Tokarev, I., Budantseva, N., Vasil'chuk, Y., Kireeva, M., Ekaykin, A., Veres, A., Aleynikov, A., Frolova, N., Tsyplenkov, A., Polukhov, A., Chalov, S., Aleshina, M., and Kornilova, E.: Glaciological, hydrological, meteorological observations and isotopes sampling results during 2007-2017 at Djankuat Glacier Station in the North Caucasus, Russia, PANGAEA, https://doi.org/10.1594/PANGAEA.894807, 2018a.

Rets, E. P., Dzhamalov, R. G., Kireeva, M. B., Frolova, N. L., Durmanov, I. N., Telegina, A. A., Telegina, E. A., and Grigoriev, V. Y.: Recent trends of river runoff in the North Caucasus, Geography, Environment, Sustainability, 11, 61-70, https://doi.org/10.24057/2071-9388-2018-11-3-61-70, 2018 b.

Schaefli, B., Hingray, B., Niggli, M., and Musy, A.: A conceptual glacio-hydrological model for high mountainous catchments, Hydrol. Earth Syst. Sci., 9, 95-109, https://doi.org/10.5194/hess9-95-2005, 2005.

Shahgedanova, M.: Climate at present and in the historical past, The Physical Geography of Northern Eurasia, edited by: Shahgedanova, M., Oxford Univ. Press, New York, 70-102, 2002.

Shahgedanova, M., Stokes, C. R., Gurney, S. D., and Popovnin, V. V.: Interactions between mass balance, atmospheric circulation and recent climate change on the Djankuat glacier, Caucasus Mountains, J. Geophys. Res., 110, D04108, https://doi.org/10.1029/2004JD005213, 2005. 
Shahgedanova, M., Nosenko, G., Kutuzov, S., Rototaeva, O., and Khromova, T.: Deglaciation of the Caucasus Mountains, Russia/Georgia, in the 21 st century observed with ASTER satellite imagery and aerial photography, The Cryosphere, 8, 2367-2379, https://doi.org/10.5194/tc-8-2367-2014, 2014.

Singh, P., Bhatnagar, N. K., and Kumar, N.: Status and problems related with mountain hydrology, National Institute of Hydrology, Roorkee, 1999.

Stokes, C., Gurney, S., Shahgedanova, M., and Popovnin, V.: Late-20th-century changes in glacier extent in the Caucasus Mountains, Russia/Georgia, J. Glaciol., 52, 99-109, https://doi.org/10.3189/172756506781828827, 2006.

Stokes, C. R., Popovnin, V., Aleynikov, A., Gurney, S. D., and Shahgedanova, M.: Recent glacier retreat in the Caucasus Mountains, Russia, and associated increase in supraglacial debris cover and supra-/proglacial lake development, Ann. Glaciol., 46, 195203, https://doi.org/10.3189/172756407782871468, 2007.

Toropov, P. A., Shestakova, A. A., and Smirnov, A. M.: Methodological aspects of heat balance components estimation on mountain glaciers, Russian Journal of Earth Sciences, ES4002, 1-9, https://doi.org/10.2205/2017ES000605, 2017.

Toropov, P. A., Aleshina, M. A., Kislov, A. V., and Semenov, V. A.: Trends of climate change in the Black sea-Caspian region in the last 30 years, Moscow University Vestnik, Series 5, Geography, 2, 67-77, 2018a.

Toropov, P. A., Shestakova A. A., Smirnov A. M., and Popovnin, V. V.: Evaluation of the components of the heat balance of the Djankuat glacier (Central Caucasus) during the period of ablation in 2007-2015, Earth's Cryosphere, 22, 42-54, 2018 b.

Viviroli, D., Archer, D. R., Buytaert, W., Fowler, H. J., Greenwood, G. B., Hamlet, A. F., Huang, Y., Koboltschnig, G., Litaor, M. I., López-Moreno, J. I., Lorentz, S., Schädler, B., Schreier, H., Schwaiger, K., Vuille, M., and Woods, R.: Climate change and mountain water resources: overview and recommendations for research, management and policy, Hydrol. Earth Syst. Sci., 15, 471-504, https://doi.org/10.5194/hess-15-471-2011, 2011.
Volodicheva, N. A. and Voitkovskiy, K. F.: Evolutsija lednikovoj sistemy Elbrusa (Evolution of Elbrus glacial system), Geogrphia, obshchestvo i okruzhayushaya sreda, Moscow, Gorodets Moskva, 1, 377-395, 2014.

Weingartner, R., Viviroli, D., and Schädler, B.: Water resources in mountain regions: a methodological approach to assess the water balance in a highland-lowland-system, Hydrol. Process., 21, 578-585, https://doi.org/10.1002/hyp.6268, 2007.

Zemp, M., Hoelzle, M., and Haeberli, W.: Six decades of glacier mass-balance observations: a review of the worldwide monitoring network, Ann. Glaciol., 50, 101-111, https://doi.org/10.3189/172756409787769591, 2009.

Zemp, M., Paul, F., Andreassen, L., M., Arino, O., Bippus, G., Bolch, T., Braithwaite, R., Braun, L., Cáceres, B. E., Casassa, G., Casey, K. A., Ceballos, C. L., Citterio, M., Delgado, H., Demuth, M., Espizua, L. E., Farokhnia, A., Fischer, A., Foppa, N., Frey, H., Fujita, K., Gärtner-Roer, I., Glowacki, P., Haeberli, W., Hagen, J. O., Hoelzle, M., Holmlund, P., Giesen, R. H., Kääb, A., Khromova, T., Kotlarski, S., Le Bris, R., Li, Z., Meier, M., Meneghel, M., Mool, P., Nussbaumer, S. U., Peduzzi, P., Plummer, S., Popovnin, V., Prinz, R., Rack, W., Rastner, P., Raup, B., Rinne, E., Seifert, F. M., Seiz, G., Severskiy, I., Shepherd, A., Sigurðsson, O., Strozzi, T., Vincent, C., Wheate, R., and Yakovlev, A.: Summary of international glacier monitoring summit, The Earth Observer, 23, 28-31, 2011.

Zemp, M., Frey, H., Gärtner-Roer, I., Nussbaumer, S. U., Hoelzle, M., Paul, F., Haeberli, W., Denzinger, F., Ahlstrøm, A. P., Anderson, B., Bajracharya, S., Baroni, C., Braun, L. N., Càceres, B. E., Casassa, G., Cobos, G., Dàvila, L. R., Delgado Granados, H., Demuth, M. N., Espizua, L., Fischer, A., Fujita, K., Gadek, B., Ghazanfar, A., Hagen, J. O., Holmlund, P., Karimi, N., Li, Z., Pelto, M., Pitte, P., Popovnin, V. V., Portocarrero, C. A., Prinz, R., Sangewar, C. V., Severskiy, I., Sigurdsson, O., Soruco, A., Usubaliev, R., and Vincent, C.: Historically unprecedented global glacier decline in the early 21 st century, J. Glaciol., 61, 745-762, https://doi.org/10.3189/2015JoG15J017, 2015. 